\title{
Les déterminants du jugement des salariés sur la RTT
}

\author{
Gilbert Cette, Nicolas Dromel et Dominique Méda*
}

Si de nombreux travaux ont déjà été consacrés aux conséquences économiques des politiques de réduction du temps de travail (RTT), notamment sur l'emploi, peu d'études ont jusqu'ici abordé les effets de ces politiques sur les conditions de travail et les conditions de vie des salariés concernés. Plusieurs études ont porté sur les attentes des salariés de la mise en place d'une réduction du temps de travail (analyses ex ante), mais beaucoup moins sur la perception et l'appréciation qu'en ont les salariés après sa réalisation (analyses $e x$ post). Cette question des facteurs de satisfaction et d'insatisfaction des salariés vis-à-vis d'une politique de réduction du temps de travail, dont ils ont vécu les effets directement, est approfondie ici en analysant les réponses à l'enquête RTT et Modes de vie, réalisée en 2001, auprès d'un échantillon de salariés à temps complet ayant connu une réduction du temps de travail dans le cadre d'un accord « Robien » ou Aubry 1 » depuis au moins un an. La prise en compte d'un grand nombre de variables permet d'évaluer l'influence de chacune d'entre elles « toutes choses égales par ailleurs».

Certaines caractéristiques semblent toujours exercer une influence sur l'appréciation des salariés vis-à-vis de la RTT et concourir au renforcement de la satisfaction. Ainsi, les femmes ayant à charge un enfant de moins de 12 ans sembleraient, toutes choses égales par ailleurs, trouver plus de satisfaction à la RTT que les autres salariés interrogés. Par ailleurs, les facteurs influençant significativement la satisfaction seraient notamment la situation sociale du salarié (appréciée conjointement par son diplôme, sa catégorie professionnelle et le revenu du ménage), la visibilité des horaires de travail et son amélioration, l'autonomie dans les horaires de travail, le fait que la durée effective correspond bien à celle qui était prévue dans l'accord, l'impact de la RTT sur la rémunération et les usages qui sont faits du temps dégagé par la RTT. Parmi ces usages, on remarque principalement l'augmentation du temps passé avec la famille (conjoint et enfants) et celui passé à se reposer ou à exercer d'autres activités domestiques, de semi-loisir ou de loisir. Le fait que le salarié ait eu avant la RTT le sentiment de manquer de temps, qu'il ait réfléchi à l'usage du temps potentiellement dégagé par la RTT, et que cet usage se soit traduit concrètement dans la réalité influencerait également sa satisfaction. Inversement, la variabilité des horaires, l'atypicité des horaires de manière générale et son augmentation, la hausse des objectifs et l'impact défavorable de la RTT sur la rémunération influenceraient négativement cette satisfaction.

\footnotetext{
* Gilbert Cette appartient à l'Université de la Méditerranée (Ceders), Nicolas Dromel à l'Université de la Méditerranée (Greqam) et Dominique Méda à la Mission Animation de la Recherche de la Dares.

Les noms et dates entre parenthèses renvoient à la bibliographie en fin d'article.

Les auteurs remercient Matthieu Bunel et Marc-Antoine Estrade (MAE-Dares), Alain Gubian (ACOSS) et Daniel Verger (Insee) pour leurs conseils et suggestions, ainsi que trois rapporteurs anonymes pour leurs remarques pertinentes, tout en restant évidemment seuls responsables des erreurs qui demeureraient.
} 
$\mathbf{L}$ e thème de la réduction du temps de travail (RTT par la suite) touche à de nombreux domaines, parmi lesquels évidemment l'économie, avec par exemple la difficile question des créations d'emplois que des politiques de RTT peuvent induire, et la sociologie, compte tenu des interactions entre les divers temps sociaux que constituent les temps de travail et « hors travail ». Ces différents domaines sont intimement liés, car si les effets économiques d'une RTT dépendent essentiellement de l'impact qu'elle a sur les coûts de production des entreprises, cet impact est influencé par les arbitrages microéconomiques entre loisirs et revenus d'activité des individus concernés. Autrement dit, les attentes et préférences des agents, quantitatifs en ce qui concerne les temps de travail et « hors travail », mais aussi, voire surtout, qualitatifs en ce qui concerne le contenu et l'articulation de ces différents temps sociaux, influencent les effets économiques de toute politique de RTT.

Si de nombreux travaux ont été consacrés au processus de négociation associé à la RTT, ou à ses effets économiques, en particulier sur l'emploi (1), les analyses des effets de la RTT sur les conditions de travail et les conditions de vie des salariés concernés, et en aval sur leur appréciation de la RTT qu'ils vivent, sont plus rares. Plusieurs études détaillées ont porté sur les attentes ex ante des salariés concernant les diverses modalités envisageables d'une RTT (2), ce qui constitue un autre type d'interrogation. Concernant les analyses ex post, depuis l'entrée en vigueur de la loi « Robien » qui a institué en 1996 un système d'allégements de charges sociales aux entreprises mettant en œuvre une RTT pour favoriser l'emploi, des sondages ou enquêtes qualitatives se sont intéressés à la « satisfaction » des salariés concernés. La CFDT a ainsi organisé, depuis 1998, diverses enquêtes rassemblées sous l'intitulé Le travail en questions, à partir d'un échantillon représentatif de 18000 salariés interrogés sur les conséquences d'accords RTT négociés dans le cadre des lois « Aubry ». L'exploitation des réponses à ces différentes enquêtes s'est faite, en général, sous la forme de tris croisés sur quelques variables et ne sont donc pas toutes choses égales par ailleurs vis-à-vis d'autres variables (3). Le Ministère de l'Emploi (Dares) a mené, quant à lui, une première enquête, en 1999, auprès de 526 salariés ayant connu au moins un an plus tôt une RTT dans le cadre d'une convention « Robien », en passant par les entreprises pour interroger les salariés, ce qui pouvait introduire un biais de sélection. Les exploitations statistiques par des approches toutes choses égales par ailleurs proposées par
Doisneau (2000) ou Cette et Diev (2002) d'une question de cette enquête sur la satisfaction globale (4) des salariés ont alors montré que les facteurs qui semblent les plus déterminants à cet égard seraient la catégorie sociale du salarié, les modalités d'application de la RTT avec par exemple les éventuelles réorganisations associées, ainsi que la visibilité et la maitrise du temps « dégagé » par la RTT (5). Ces résultats confirmaient, d'ailleurs, les enseignements de quelques études qualitatives antérieures de nature sociologique réalisées auprès d'échantillons très réduits de salariés (6).

Cette question des facteurs de satisfaction et d'insatisfaction des salariés vis-à-vis d'une RTT qui les concerne directement est approfondie ici en analysant les réponses à l'enquête $R T T$ et Modes de vie conçue par la Dares et réalisée entre la fin de l'année 2000 et le début de l'année 2001 auprès d'un échantillon de 1618 salariés représentatifs des salariés ayant connu une RTT au moins un an plus tôt dans le cadre des dispositifs « Robien » et « Aubry 1 » (cf. encadré). Ici encore, comme dans certaines analyses précédentes, le recul d'une année au moins permet de bénéficier d'une appréciation sans doute plus robuste de la part des salariés interrogés. Ces derniers ont été interrogés en face-à-face à leur domicile sans que leurs employeurs soient sollicités, les adresses provenant directement des DADS 1999 (Données Annuelles de Données Sociales) des entreprises qui avaient mis en œuvre un accord de RTT avant novembre 1999. Afin de caractériser au mieux les facteurs de satisfaction et d'insatisfaction, on s'efforce de prendre en compte simultanément un grand nombre de variables renseignées par l'enquête pour évaluer l'influence de chacune d'entre elles, via une analyse toutes choses égales par ailleurs par l'estimation de modèles logistiques. Toutefois, si une telle approche aboutit à des enseignements riches, elle ne peut prétendre fournir des éléments d'évaluation des dispositifs de RTT concernés («Robien » et « Aubry 1 »,cf. encadré).

1. Voir par exemple sur ces aspects les numéros spéciaux de Travail et Emploi (2000a et 2000b) et d'Économie Internationale (2000)

2. Par exemple Boulin, Cette et Verger (1998a et 1998b).

3. Les résultats de ces exploitations ont été publiés dans CFDT (1999), CFDT (2001) et Romanovski (2002).

4. Cette question y était ainsi libellée : "d'une manière générale, diriez-vous que vous êtes tout à fait satisfait, plutôt satisfait, plutôt pas satisfait ou pas satisfait du tout de la répartition actuelle de votre temps de travail ? ".

5. Les résultats de cette enquête ont également fait l'objet de diverses exploitations sous forme de tris croisés, par exemple dans MES (1999) et CGP (2001).

6. Voir par exemple Boulin, Lallement, Lefèvre et Silvéra (1998). 
L'approche toutes choses égales par ailleurs permet cependant d'aboutir à des résultats assez structurels et peu contingents au fait que les salariés enquêtés sont concernés par des RTT engagées avant la fin 1999. En d'autres termes, si les entreprises employant ces salariés présentent peut-être, par rapport à toutes les entreprises passées aux 35 heures et plus encore par rapport à l'ensemble des entreprises, des caractéristiques particulières (biais de sélection des entreprises), on fait l'hypothèse que cela n'affecte pas les résultats de l'analyse qui dégage, toutes choses égales par ailleurs, la corrélation statistique entre diverses caractéris- tiques des salariés et des entreprises et la satisfaction (positive ou négative) apportée par les 35 heures. Si les caractéristiques des entreprises ou des salariés peuvent présenter des éléments de spécificité, les corrélations dégagées par l'exploitation de l'enquête sont sans doute structurelles, même s'il faut rester prudent quant à une généralisation des résultats à tous les cas de réduction du temps de travail en raison de possibles biais de sélection (caractéristiques non observées de l'entreprise susceptibles d'affecter la corrélation entre caractéristiques non observées et satisfaction).

Encadré

\section{LES DISPOSITIFS LÉGISLATIFS DE RÉDUCTION DU TEMPS DE TRAVAIL (RTT) CONCERNÉS DANS L'ENQUÊTE RTT ET MODES DE VIE}

Du fait de sa date de réalisation (fin 2000 et début 2001) et de la condition d'un accord RTT antérieur d'au moins un an, l'enquête RTT et Modes de vie ne concerne que des salariés ayant réduit leur durée de travail avant la loi «Aubry 2 » du 19 janvier 2000, dans le cadre de dispositifs « Robien » ou « Aubry 1 ».

La loi n`96-502 du 11 juin 1996, dite loi «Robien », institue un système d'allégements de cotisations sociales pour les entreprises qui réalisent une RTT collective, en respectant certains engagements en termes d'emplois. Les aides sont conditionnées à la signature d'une convention avec l'État, faisant suite à un accord signé entre partenaires sociaux. Pendant les deux années d'application de cette loi, environ 3000 conventions ont été signées, réduisant le temps de travail de 280000 salariés et prévoyant 33000 créations ou maintiens d'emplois.

Ce dispositif est abrogé par la loi $n^{\circ} 98-461$ du 13 juin 1998, dite première loi «Aubry » ou loi « Aubry 1 », qui (entre autres nombreuses dispositions) fixe la durée légale du travail à 35 heures hebdomadaires au $1 \mathrm{er}$ janvier 2000 pour les entreprises de plus de 20 salariés au 1er janvier 2002 pour les autres. La loi «Aubry 1 » institue un nouveau dispositif d'allégements de cotisations sociales incitant les entreprises à négocier une RTT avant la baisse de la durée légale. Ces allégements sont également conditionnés à la signature d'une convention avec l'État, faisant suite à celle d'un accord entre partenaires sociaux.

\section{Des dispositifs d'allégements de charges sociales} pour maintenir l'emploi

Le dispositif «Robien » (resp. «Aubry 1 ») comprend un volet "offensif» dans lequel un allégement des cotisations sociales est subordonné à l'augmentation des effectifs de $10 \%$ (resp. de $6 \%$ ) en cas d'une RTT de $10 \%$; de $15 \%$ (resp. de $9 \%$ ) en cas d'une RTT de $15 \%$ ou plus. Le niveau d'emploi atteint doit être maintenu pendant au moins deux ans. Les deux dispo- sitifs comprennent un volet "défensif ", dans lesquels un allégement bénéficie aux unités qui réduisent la durée du travail afin d'éviter des licenciements prévus dans le cadre d'une procédure de licenciement économique, et qui s'engagent à maintenir les effectifs couverts pour une durée fixée par la convention. Les engagements en termes de maintien de l'emploi sont dans le dispositif «Robien » (resp. «Aubry 1 ») de $10 \%$ des effectifs concernés (resp. $6 \%$ ) en cas d'une RTT de $10 \%$; de $15 \%$ (resp. $9 \%$ ) lorsque la RTT est de $15 \%$

Entre autres différences, les dispositifs « Robien » et "Aubry 1 » se distinguent par le fait que les allégements de charges sociales sont proportionnels au salaire dans le premier et forfaitaires dans le second, et que le second est instauré avec la perspective d'une baisse certaine de la durée légale du travail, ce qui n'était pas le cas du premier.

Par ailleurs, après l'application de la loi « Aubry 1 », un certain nombre d'entreprises ont réduit leur durée collective de travail sans aide incitative avant novembre 1999, en anticipant la nouvelle durée hebdomadaire légale de 35 heures. C'est le cas, d'une part d'entreprises du secteur privé qui ne sont pas entrées dans le dispositif d'incitation parce qu'elles ne voulaient ou ne pouvaient pas s'engager sur les conditions minimales d'octroi de l'aide «Aubry 1 ", et d'autre part de grandes entreprises publiques qui ont réduit leur durée du travail sans pouvoir bénéficier d'allégement de cotisations sociales (entreprises non éligibles). Ces dernières ne font pas partie du champ de l'enquête.

Certaines formes de RTT qui ont été rendues possibles par la loi «Aubry 2 » ne sont pas présentes (forfait jours, définition d'une durée annuelle, etc.) ou ne concernent que les entreprises ayant réduit le temps de travail sans aide incitative (modification du mode de décompte de la durée).

Pour plus de détails sur ces dispositifs, on pourra se reporter à (Commissariat général du Plan, 2001). 


\section{Les résultats de l'enquête RTT et Modes de vie}

L 'enquête RTT et Modes de vie avait pour objectif principal de mettre en évidence les principaux changements intervenus dans la vie des salariés à temps complet ayant connu une RTT depuis au moins un an (7) (cf. annexe 1 pour une présentation détaillée de l'enquête). Pour éviter d'éventuels biais liés à une acceptation ou non-acceptation d'un questionnement de leurs salariés par les entreprises, un échantillon représentatif de la population cible a été constitué grâce aux DADS, les salariés étant directement contactés pour être interrogés en face-àface dans le cadre d'un entretien d'environ une heure. Réalisée entre novembre 2000 et janvier 2001, elle concerne des salariés à temps complet passés à 35 heures avant la fin 1999 dans le cadre des dispositifs « Robien » ou « Aubry 1 » (cf. encadré).

L'enquête contient à la fois des questions subjectives (par exemple sur l'impression de manquer de temps avant la RTT ou sur la perception des différents changements liés à celle-ci) mais tente aussi d'objectiver et de vérifier les déclarations des salariés au moyen d'un certain nombre de données concrètes sur le temps de travail, les modalités de détermination des horaires, les revenus, les activités exercées pendant le temps dégagé par la RTT. Le questionnement vise d'abord à déterminer les conditions pratiques de la négociation de l'accord RTT (et la façon dont les salariés ont été plus ou moins associés au processus) et à mesurer la réalité de la RTT, avant de rentrer dans le détail des changements intervenus, à différentes échelles temporelles, au niveau des horaires de travail, des conditions de travail, de la vie familiale, des tâches domestiques, des loisirs et de la rémunération. Le questionnaire se terminait par une question dite de bilan, censée faire la synthèse des différents éléments qui ont été passés en revue par le salarié et qui concerne conjointement la vie au travail et la vie «hors travail»: «globalement, diriez-vous que les effets de la RTT sur votre vie quotidienne (aussi bien au travail qu'en dehors du travail) ont été plutôt : 1. Dans le sens d'une amélioration; 2. Dans le sens d'une dégradation; 3.Cela n'a rien changé ». Ce sont les réponses à cette question qui indiquent la satisfaction ou l'insatisfaction apportée par la RTT. Cette question est considérée comme conduisant le salarié à indiquer la satisfaction (ou l'insatisfaction) globalement induite par la RTT via les changements intervenus dans sa vie au travail et dans sa vie « hors travail ».
Les principaux résultats généraux de l'exploitation de cette enquête par tris croisés ont fait l'objet de diverses présentations (Estrade, Méda et Orain, 2001 ; Estrade et Méda, 2002 ; Estrade et Ulrich, 2002 ; Méda et Delteil, 2002 ; Méda et Orain, 2002) et ne sont que brièvement résumés ici.

\section{Une modification des rythmes et des usages du temps}

Du point de vue des conséquences financières du passage à la RTT, $12 \%$ des salariés ont connu une baisse de leur salaire, $56 \%$ un gel ou une modération de leur rémunération et le passage n'a entraîné aucun impact sur la rémunération pour $32 \%$ d'entre eux.

Si elle n'a pas bouleversé l'organisation des horaires journaliers, la RTT a entraîné une modification des rythmes et des usages du temps sur la semaine, voire sur des périodes plus longues, notamment parce que les modalités de RTT les plus fréquentes ont été la possibilité de disposer de demi-journées ou journées de manière régulière (36\% des salariés), des jours de congé ou jours RTT supplémentaires (35\%) et la modulation $(20 \%)$, le raccourcissement de l'horaire quotidien ne concernant que moins d'un quart des salariés.

Les usages principaux du temps dégagé par la RTT sont le bricolage et le jardinage, le repos et le temps passé avec les enfants (les hommes avec enfants de moins de douze ans sont $52 \%$ à déclarer passer plus de temps avec leurs enfants depuis la RTT et les femmes avec enfants de moins de douze ans $63 \%$, les parents d'enfants de moins de douze ans étant aussi $37 \%$ à déclarer que la conciliation de la vie professionnelle et de la vie familiale s'est améliorée depuis la RTT). En revanche, la RTT n'a pas remis en cause la division traditionnelle des rôles dans le couple et la prise en charge principalement par les femmes des tâches domestiques, même si celles-ci sont réalisées à des moments permettant de libérer davantage le week-end de ces tâches.

7. Les chiffres et pourcentages présentés dans cette partie, issus des premiers résultats généraux de l'enquête, ne sont pas directement comparables avec ceux du tableau $B$ en annexe 1 (dénombrement des modalités de réponses aux questions constituant les variables explicatives de l'analyse). Cependant, il n'apparaît pas d'incohérence notable entre ces deux sources d'information. Les écarts pouvant toutefois subsister proviennent des différences au niveau de la construction des variables retenues selon les études, elles-mêmes dépendantes des typologies et concepts arrêtés par les auteurs. 


\section{En moyenne, un sentiment d'amélioration} de la vie quotidienne...

Un certain nombre de tris croisés ont été aussi consacrés à la caractérisation et aux facteurs associés aux réponses données à la question concernant l'amélioration (ou la dégradation) de la vie quotidienne, à la suite d'une RTT, notamment dans Estrade, Méda et Orain (2001) et Méda et Orain (2002). Ils visent, notamment, à mettre en évidence dans quelle mesure les réponses étaient différentes selon la catégorie professionnelle, le sexe, la configuration familiale des salariés, le dispositif de RTT, les modalités concrètes de négociation et d'application de l'accord, leur rémunération, l'effectivité et l'usage d'un surcroît de temps non travaillé.
Le sentiment d'amélioration de la vie quotidienne est, en moyenne, plutôt positif puisque $59 \%$ des salariés répondent que les effets de la RTT sur leur vie quotidienne ont plutôt été «dans le sens d'une amélioration», $13 \%$ plutôt «dans le sens d'une dégradation», $28 \%$ considérant que «cela n'a rien changé » (cf. tableau 1). Cette moyenne recouvre cependant de fortes disparités, la perception d'une amélioration est, par exemple, nettement plus fréquente pour les femmes ayant à charge un enfant de moins de 12 ans (cf. tableau 2). Méda et Orain (2002) ont avancé l'hypothèse que si les femmes travaillant à temps complet ayant des enfants de moins de douze ans et a fortiori de moins de trois ans étaient plus satisfaites de la RTT que la moyenne des salariés (71\%

Tableau 1

Sentiment d'une amélioration ou d'une dégradation consécutive à la RTT selon le sexe et la CS

\begin{tabular}{|c|c|c|c|c|}
\hline & & $\begin{array}{r}\text { Globalement, diriez-vc } \\
\text { aussi bien }\end{array}$ & $\begin{array}{l}\text { que les effets de la RT } \\
\text { travail qu'en dehors du }\end{array}$ & $\begin{array}{l}\text { Ir votre vie quotidienne, } \\
\text { lail ont été... }\end{array}$ \\
\hline Sexe de l'enquêté(e) & $\begin{array}{c}\text { Catégorie professionnelle } \\
\text { de l'enquêté(e) }\end{array}$ & $\begin{array}{l}\text {... plutôt dans le sens } \\
\text { d'une amélioration? }\end{array}$ & $\begin{array}{l}\text {... plutôt dans le sens } \\
\text { d'une dégradation? }\end{array}$ & Cela n'a rien changé \\
\hline & $\begin{array}{l}\text { Ensemble } \\
\text { dont : }\end{array}$ & 58,4 & 12,7 & 28,9 \\
\hline Homme & Cadre & 64,9 & 6,7 & 28,4 \\
\hline & Profession intermédiaire & 57,3 & 13,4 & 29,3 \\
\hline & Employé ou ouvrier qualifié & 56,5 & 14,3 & 29,2 \\
\hline & Employé ou ouvrier non qualifié & 57,2 & 15,4 & 27,4 \\
\hline & $\begin{array}{l}\text { Ensemble } \\
\text { dont : }\end{array}$ & 61,0 & 13,0 & 26,0 \\
\hline Femme & Cadre & 72,5 & 8,0 & 19,5 \\
\hline & Profession intermédiaire & 73,3 & 7,4 & 19,3 \\
\hline & Employée ou ouvrière qualifiée & 60,4 & 14,0 & 25,6 \\
\hline & $\begin{array}{l}\text { Employée ou ouvrière non } \\
\text { qualifiée }\end{array}$ & 40,2 & 20,3 & 39,5 \\
\hline & Ensemble & 59,2 & 12,8 & 28,0 \\
\hline
\end{tabular}

Lecture : 64,9\% des cadres de sexe masculin ont constaté une amélioration de leur vie quotidienne depuis la mise en œuvre de la RTT. Source des données de base : enquête RTT et Modes de vie, Dares.

Tableau 2

Sentiment d'une amélioration ou d'une dégradation consécutive à la RTT selon le type de foyer

\begin{tabular}{|c|c|c|c|}
\hline \multirow{2}{*}{ Type de foyer } & \multicolumn{3}{|c|}{$\begin{array}{c}\text { Globalement, diriez-vous que les effets de la RTT } \\
\text { sur votre vie quotidienne, aussi bien au travail } \\
\text { qu'en dehors du travail ont été... }\end{array}$} \\
\hline & $\begin{array}{l}\text {... plutôt dans le sens } \\
\text { d'une amélioration }\end{array}$ & $\begin{array}{l}\text {... plutôt dans le sens } \\
\text { d'une dégradation }\end{array}$ & Cela n'a rien changé \\
\hline Ensemble de la population enquêtée & 59,2 & 12,8 & 28,0 \\
\hline $\begin{array}{l}\text { Sous-populations des foyers avec enfant de moins de } 12 \text { ans } \\
\text { dont : }\end{array}$ & 62,7 & 9,8 & 27,5 \\
\hline Femme avec enfant de moins de 12 ans & 71,0 & 4,8 & 24,2 \\
\hline Homme avec enfant de moins de 12 ans & 60,0 & 11,4 & 28,6 \\
\hline
\end{tabular}

Lecture : 71 \% des femmes avec enfant de moins de 12 ans ont constaté une amélioration de leur vie quotidienne depuis la mise en œuvre de la RTT.

Source des données de base : enquête RTT et Modes de vie, Dares. 
contre $59 \%$ ), c'est sans doute parce que, ayant à combiner travail à temps plein et prise en charge des tâches familiales et domestiques, elles ressentaient plus que la moyenne des salariés la pression du temps ou le sentiment d'en manquer (ce que confirment les données puisque $57 \%$ des femmes de l'échantillon avec enfants de moins de douze ans déclarent qu'elles avaient l'impression de manquer souvent ou toujours de temps avant la RTT, alors que la moyenne des personnes interrogées est de $39 \%$ ) et ressentaient sans doute, plus que les autres, la nécessité de disposer de plus de temps.

\section{... mais un bilan plus nuancé sur les conditions de travail}

Si le sentiment d'amélioration, synthèse des effets ressentis sur la vie au travail et en dehors du travail, est positif, le bilan concernant les conditions de travail est plus nuancé puisqu'un petit quart des salariés seulement déclarent avoir connu une amélioration de celles-ci, un gros quart une dégradation, alors que $46 \%$ trouvent la situation inchangée. L'intensification du travail et l'exigence accrue de polyvalence (près d'un salarié sur deux) vont de pair avec le sentiment d'une dégradation des conditions de travail, même si ceux qui ressentent le plus cette intensification sont des professions intermédiaires et des cadres dont le jugement sur l'évolution des conditions de travail est plus positif que les autres catégories.

Le sentiment d'amélioration de la vie quotidienne est plus fréquent chez les cadres que chez les employés et ouvriers non qualifiés (8) et cette fréquence est croissante avec le diplôme, le revenu, le caractère urbain de l'habitat et la présence dans le foyer d'enfants de moins de douze ans. Il est également plus fréquent dans les entreprises de 0 à 49 salariés que dans les autres, ainsi que dans les secteurs caractérisés par une organisation du temps de travail régulière.

Ce sentiment d'amélioration est aussi lié au « régime» sous lequel l'accord de RTT a été passé, la satisfaction la plus fréquente étant associée à des accords passés avec des aides incitatives de l'État et le sentiment de dégradation plus fréquent dans les accords sans aide incitative. Ainsi, $65 \%$ des salariés se trouvant dans des entreprises ayant réduit leur durée du travail dans le cadre du dispositif incitatif «Aubry 1 » font état d'une amélioration de leur vie quotidienne, alors que $43 \%$ des salariés tra- vaillant dans des entreprises ayant réduit le temps de travail sans aide incitative considèrent que la RTT n'a eu aucun effet sur leur vie quotidienne. Cela peut s'expliquer par le fait que, pour obtenir les aides, les entreprises devaient présenter des accords respectant un certain nombre de conditions, portant notamment sur le maintien du mode de décompte du temps de travail (pauses identiques par exemple) et des créations d'emplois.

Le sentiment d'amélioration est également plus fréquent lorsque les salariés ont été consultés, lorsque leurs contraintes personnelles ont été prises en compte et lorsque les termes de l'accord sur la durée effective du travail ont été respectés : un quart des salariés fait état d'une durée du travail effective supérieure à celle prévue dans l'accord, un salarié sur dix bénéficiant - au moins partiellement - d'une compensation en repos, tandis que le paiement du dépassement d'horaire en heures supplémentaires est marginal. Le dépassement de la durée prévue est majoritaire chez les cadres, le plus souvent sans compensation. Pour toutes les catégories socioprofessionnelles, le sentiment global d'une amélioration est moins répandu lorsque la durée effective dépasse celle prévue dans l'accord. Cependant, cette corrélation est plus sensible chez les non-qualifiés que chez les cadres. Chez ces derniers, le dépassement des horaires était déjà fréquent avant la RTT.

La fréquence du sentiment d'amélioration est croissante avec l'augmentation des effectifs dans l'unité de travail (service, atelier, ligne de production) et diffère selon les conséquences salariales de l'accord : quand il y a baisse de la rémunération, le bilan global est plus négatif, même si cette baisse a fréquemment eu lieu en contrepartie de licenciements évités. L'impact du gel des salaires sur la vie quotidienne est, en revanche, plus neutre.

Le sentiment d'amélioration est aussi lié aux modalités d'application de la RTT. Les salariés faisant état d'un sentiment global d'amélioration de leurs conditions de vie quotidienne (au travail et en dehors) sont ceux qui ont bénéficié d'une demi-journée ou d'une journée à prendre régulièrement, ou de jours de congés supplémentaires. Les salariés dont le temps de travail est modulé font moins état d'une amélioration de leurs conditions de vie et de travail. En

8. Le contenu des catégories " qualifiés » et « non-qualifiés » est donné dans l'annexe 1. 
effet, dans le cas des accords de modulation, les périodes basses peuvent inclure des jours de RTT déterminés par l'employeur.

La fréquence de la satisfaction globale est enfin croissante avec la perception d'une amélioration des conditions de travail, comme avec la possibilité d'avoir plus d'activités « hors travail» depuis la RTT. Et ce sentiment d'amélioration est plus fréquent quand les salariés indiquaient qu'ils avaient le sentiment de manquer de temps avant la RTT.

Ces résultats généraux ont été obtenus par des tris croisés et peuvent, pour certains d'entre eux, refléter des simultanéités sans réelle relation de cause à effet. Les tris croisés ont pour principal défaut de mêler les «effets propres» d'une dimension et les «effets indirects » d'autres variables. L'approche toutes choses égales par ailleurs remédie à cet inconvénient.

\section{Une analyse toutes choses égales par ailleurs de la satisfaction des salariés}

L estimation de modèles logistiques permet de caractériser la relation statistique, toutes choses égales par ailleurs, entre de nombreuses variables et la variable d'intérêt, ici la satisfaction que les salariés trouvent a posteriori à l'égard de la RTT mise en œuvre au moins un an plus tôt (9). Ces estimations ne visent pas à appréhender des comportements moyens comme cela a été fait précédemment. Pour cette raison, les estimations sont réalisées sur l'échantillon brut de l'enquête hors redressements (10) (cf. annexe 2).

Dans ces estimations logistiques, la variable expliquée se rapporte à la satisfaction que les salariés trouvent ou non à la RTT. C'est ici la réponse à la question de bilan du questionnaire sur la satisfaction globale citée plus haut. La formulation assez générale de cette question permet de prendre en compte avec recul les diverses dimensions de la satisfaction tirée par les salariés du processus de RTT qu'ils ont connu (11).

\section{Cinq ensembles de variables explicatives}

Les variables explicatives retenues sont décomposées en cinq ensembles regroupant :
1. Des variables d'état concernant le salarié (sexe et présence d'enfant(s), âge, catégorie professionnelle, etc.) et l'entreprise (nombre de salariés) ;

2. Des variables caractérisant l'accord de RTT : RTT aidée ou non, dispositif «Robien» ou « Aubry 1 », consultation ou non des salariés et par qui, avant l'accord de RTT ;

3. Des variables caractérisant les modifications induites par la RTT pour le salarié interrogé. Ces variables concernent les modalités d'application de la RTT (journées plus courtes, demijournées ou journées RTT, etc.), la variabilité et la visibilité des horaires, l'autonomie dans les horaires de travail, l'atypicité des horaires de travail, les conditions de travail, l'impact de la RTT sur la rémunération, etc.

4. Des variables caractérisant l'usage par les salariés du temps dégagé par la RTT: temps passé en famille, activités domestiques, repos, loisirs, etc.

5. Des variables correspondant à des questions plus «subjectives » concernant la période précédant la RTT. Ces variables caractérisent l'idée que les salariés avaient, avant l'application de la RTT, de l'utilisation du temps libre qui en découlerait et l'impression de manquer ou non de temps. Pour autant, les réponses aux questions correspondantes sont une reconstitution, par les salariés, de l'idée qu'ils se faisaient de la RTT avant qu'elle ne les concerne personnellement, c'est-à-dire au moins un an avant l'enquête. Outre les éléments d'oubli de leurs véritables idées préalables à la RTT, ce qu'ils en expriment a posteriori peut être influencé par la pratique même, assez longue de surcroît, de cette RTT. Il convient donc d'être particulièrement prudent dans l'analyse des résultats obtenus sur ces variables.

Dans les estimations réalisées pour chaque variable explicative, l'une des modalités est retenue comme référence à laquelle s'oppose(nt) l'autre (ou les autres). Pour les variables explicatives pouvant prendre diverses modalités (par exemple la catégorie professionnelle), l'une des modalités extrêmes, généralement la

9. L'annexe 2 fournit quelques indications résumées concernant les principes généraux des estimations logistiques.

10. Lollivier, Marpsat et Verger (1996, p. 49) soulignent d'ailleurs que "lorsque les poids sont peu dispersés, les résultats des régressions pondérées et non pondérées au niveau des coefficients sont peu différents et le choix est donc de peu d'importance ".

11. L'annexe 1 donne un dénombrement des diverses modalités de réponses à cette question. 
plus faible (ici l'absence de diplôme), est retenue comme la référence à laquelle s'oppose chacune des autres modalités. Enfin, pour les variables pouvant être renseignées de façon continue (par exemple le revenu mensuel du ménage), diverses modalités regroupant des intervalles de réponses possibles ont été construites de façon empirique, la modalité de référence retenue étant également l'une des modalités extrêmes, généralement la plus faible (ici moins de 10000 francs de revenu mensuel).

Certaines variables explicatives initialement utilisées ont été écartées dans les estimations finalement retenues, car elles n'interviennent jamais de façon significative. Il en est ainsi, par exemple, du secteur de l'entreprise employant le salarié interrogé, du contrôle ou non des horaires de travail, des modifications éventuelles dans le calendrier d'accomplissement des activités domestiques et du degré de commodité des horaires de travail. A contrario, d'autres variables explicatives tirées de l'enquête auraient également pu être retenues dans l'analyse. Cependant, compte tenu de leurs diverses modalités possibles, celles qui ont été choisies correspondent déjà, quand elles sont toutes présentes, à plus de 80 variables explicatives binaires dans les estimations logistiques. Le choix de celles retenues a été réalisé soit en fonction d'une logique habituelle aux estimations sur échantillon d'individus (pour les variables d'état par exemple), soit en visant à couvrir les différents domaines pour lesquelles des analyses antérieures avaient mis en valeur certaines influences, soit en fonction de premiers résultats d'estimations obtenus. Par ailleurs, le commentaire qui suit est parfois volontairement prudent pour dégager l'effet de certaines variables explicatives pouvant être liées à d'autres. Un exemple en est fourni par les trois variables de revenu mensuel, de diplôme et de catégorie professionnelle - fortement corrélées - et dont les effets doivent, en conséquence, être appréciés globalement plutôt qu'individuellement comme l'effet de la catégorie sociale du salarié.

\section{Estimer globalement la satisfaction ou l'insatisfaction des salariés}

Les estimations réalisées cherchent à expliquer par quels aspects et quels changements la RTT apporte globalement une satisfaction ou une insatisfaction aux salariés interrogés. Cet effet de la RTT résulte ici d'une agrégation de multiples micro-dimensions, prises en compte par un vaste ensemble de variables explicatives. Cer- taines de ces variables sont indépendantes de la RTT (variables d'état ou variables caractérisant l'accord de RTT par exemple) tandis que d'autres résultent d'un « libre choix » associé à la RTT (usage du temps « libéré » par exemple). Une approche plus structurelle que celle retenue aurait consisté à « expliquer » l'expression concrète de ces « libres choix » associés à la RTT par des variables indépendantes de cette dernière, pour caractériser plus explicitement la fonction d'utilité du salarié, cette fonction articulant des éléments ressortant tant de la sphère du travail que de la sphère « hors travail », les deux étant affectées par la RTT. Ici, l'objectif est plus réduit et se limite à caractériser les facteurs influençant positivement ou négativement la satisfaction globale apportée au salarié par la RTT. Le risque de non-exogénéité de certaines variables prises en compte est bien réel, par exemple pour celles concernant les usages du temps « libéré », et les commentaires proposés sont, en conséquence, prudents. Pour autant, l'ajout successif des différents ensembles de variables explicatives a permis de s'assurer d'une certaine robustesse des résultats et de mettre en évidence plus précisément les instabilités - ou leur manque de robustesse - pour en proposer une interprétation spécifique.

Les estimations logistiques ont été réalisées de façon dichotomique, en opposant pour la variable expliquée l'amélioration à l'absence de changement et à la dégradation, ou bien en opposant l'amélioration et l'absence de changement à la dégradation. Elles ont aussi été réalisées de façon polytomique ordonnée ou non ordonnée, en distinguant les trois modalités de réponse à la question concernant la variable expliquée. Les résultats obtenus avec ces diverses options de traitement de la variable expliquée sont très proches et cohérents entre eux.

Par ailleurs, pour chacune des différentes options de traitement de la variable expliquée, les estimations logistiques ont été réalisées en ajoutant successivement chacun des cinq ensembles de variables explicatives présentés plus haut afin de s'assurer de la robustesse des résultats obtenus vis-à-vis des relations éventuelles entre ces différents ensembles de variables explicatives. De fait, les cas de changements significatifs du coefficient d'une variable d'un ensemble lors de l'ajout d'un autre ensemble de variables sont rares et seront commentés. Les résultats obtenus apparaissent donc globalement robustes au nombre d'ensemble de variables explicatives. 
De nombreuses variables paraissent influencer la satisfaction tirée par les salariés de la RTT (12). Rigoureusement, l'analyse réalisée permet de caractériser une relation statistique « toutes choses égales par ailleurs » entre diverses variables et la fréquence de satisfaction apportée aux salariés par la RTT. Par commodité, on écrit par la suite indifféremment que la satisfaction est plus forte ou plus fréquente, ou à l'inverse qu'elle est plus faible ou moins fréquente, pour telle(s) ou telle(s) modalité(s) d'une variable explicative.

\section{Les variables d'état du salarié}

Concernant les variables d'état du salarié, la satisfaction serait plus fréquente quand le salarié interrogé est une femme (ici travaillant à temps complet) ayant à charge un enfant de moins de 12 ans (cf. tableau 3). Dans les premières estimations réalisées, le sexe et la présence d'enfants constituaient des variables distinctes dont aucune des modalités n'influençait significativement la satisfaction du salarié interrogé. Ainsi, globalement, les femmes et les hommes ne se distinguent pas - toutes choses égales par ailleurs - par une fréquence de satisfaction différente. De même, la présence d'enfant(s) n'influence pas - toutes choses égales par ailleurs - significativement la satisfaction du salarié (13). Mais le croisement des modalités de ces deux variables (sexe et présence d'enfant) permet de dégager des facteurs de satisfaction statistiquement significatifs (14). Ainsi, en croisant le sexe et la présence ou non d'enfant(s) de moins de 12 ans, les femmes ayant à charge un enfant de moins de 12 ans sont significativement plus souvent satisfaites de la RTT - toutes choses égales par ailleurs - que les trois autres catégories entre lesquelles la fréquence de satisfaction n'est pas significativement différente. Ce résultat, qui rejoint celui commenté par Méda et Orain (2002) sur la base de tris croisés, s'explique assez facilement par la division dans le couple de la charge du travail domestique occasionnée par la présence d'enfant(s). Les mères, assurant en général une plus forte part de cette charge que les pères, trouvent plus de satisfaction que ces derniers dans une RTT qui leur facilite la conciliation entre travail et activités familiales et domestiques.

La satisfaction serait moins fréquente quand le salarié a un conjoint que lorsqu'il n'en a pas. De fait, lorsque les estimations sont réalisées sur des variables explicatives n'incluant pas celles regroupées dans l'ensemble « usages du temps dégagé par la RTT », la présence d'un conjoint n'est associée à une plus faible fréquence de satisfaction que dans le cas où le conjoint travaille à temps complet, et plus particulièrement lorsqu'il connaît une RTT avec modulation (ce résultat est commenté infra de façon plus détaillée avec l'analyse des variables d'usage du temps).

En revanche, la satisfaction serait croissante avec la catégorie sociale du salarié, appréciée simultanément par le niveau de diplôme, la catégorie professionnelle et le revenu mensuel. Cette influence de la catégorie sociale du salarié est indépendante de l'impact de la RTT sur sa rémunération ou sur les conditions de travail, ces aspects étant pris en compte par d'autres variables spécifiques. Elle signifie ici que - toutes choses égales par ailleurs - le gain de temps libre et les nouvelles occupations rendues possibles par la RTT procurent d'autant plus de satisfaction au salarié que sa catégorie sociale est élevée, ce qui peut recouvrir de nombreuses dimensions culturelles.

Elle serait également croissante avec le caractère urbain de l'habitat, peut-être en raison des utilisations possibles du temps libéré souvent plus nombreuses dans les plus grandes agglomérations. En revanche, elle serait indépendante de son temps de transport ainsi que du secteur d'activité et des effectifs de l'entreprise qui l'emploie. Que la satisfaction soit indépendante de la présence et du nombre d'enfant(s) n'empêche pas, on le verra plus loin, qu'elle soit plus élevée quand une partie du temps dégagé par la RTT est passé en famille.

\section{Les variables caractérisant l'accord RTT}

La satisfaction serait plus fréquente dans le cas où l'accord de RTT a donné lieu à une aide incitative, quel que soit le dispositif («Robien » ou «Aubry $1 »$, défensif ou offensif) (cf. tableau 3).

12. Les résultats détaillés des diverses estimations réalisées sont présentés en annexe 2.

13. Le fait que les variables non croisées ne ressortent pas significativement-alors que les femmes ayant à charge un enfant de 12 ans sont plus souvent satisfaites de la RTT que les autres croisements de ces deux variables (sexe et présence d'enfant(s)) - s'explique statistiquement par le faible nombre de salariées concernées : moins de $10 \%$ de l'échantillon global (cf. tableau $A$ en annexe 1). Signalons que le fait de croiser les deux variables sexe et présence d'enfant(s) ne modifie que très marginalement les coefficients (et leur significativité) des modalités des autres variables.

14. C'est à une remarque de Matthieu Bunel, que nous tenons à remercier, que nous devons d'avoir croisé ces variables pour dégager ainsi l'effet significatif ici mentionné. 
Cela est à mettre en rapport avec le fait que les entreprises demandant l'aide de l'État devaient respecter strictement un certain nombre de conditions comme de ne pas changer le mode de décompte du temps de travail (les pauses par exemple).

Elle serait aussi plus fréquente lorsque l'accord de RTT a donné lieu à une consultation, via la direction de l'entreprise ou via un groupe de travail. Ce résultat n'est cependant pas robuste : la consultation par la direction ou par un groupe de travail n'apparaît plus significative dès lors que sont également intégrés comme facteurs explicatifs de la satisfaction des salariés, respectivement les modifications induites par la RTT et les usages du temps dégagé par la RTT. La fréquence de satisfaction n'apparaît jamais statistiquement liée à l'existence d'une consultation par les syndicats ou par les élus du personnel.

\section{Les variables concernant les modifications induites par la RTT}

La satisfaction serait plus fréquente lorsqu'une (ou plusieurs) modalité de RTT est clairement identifiée (retenue) parmi les modalités suivantes: journées de travail plus courtes, demi-journées ou journées RTT non travaillées, modulation/annualisation (cf. tableau 3). En revanche, la satisfaction ne serait pas significativement influencée par le fait que la RTT prenne la forme de compte épargne-temps, capital temps ou compensation.

Elle serait croissante avec la visibilité des horaires de travail mais décroissante avec la variabilité des horaires. Elle serait également croissante avec l'évolution de la visibilité induite par la mise en œuvre de la RTT (15) et avec l'autonomie du salarié dans la pratique des horaires de travail mais non significativement en relation avec l'augmentation de cette autonomie.

Moins fréquente lorsque les horaires de travail sont atypiques, tout particulièrement pour la plage horaire allant de 20 heures à 5 heures et, à moindre titre, de 5 heures à 7 heures, elle serait, par contre, indépendante des horaires de travail entre 18 heures et 20 heures. La satisfaction serait également plus faible lorsque l'atypicité des horaires a augmenté avec l'application de la RTT mais plus fréquente lorsque les horaires de travail correspondent à ceux prévus dans l'accord RTT que lorsqu'ils n'y correspondent pas.
La satisfaction serait aussi moins fréquente lorsque les objectifs de travail ont augmenté à la suite de la RTT. Par contre, elle serait indépendante d'un éventuel raccourcissement des délais de réalisation des objectifs et d'autant moins fréquente que l'impact de la RTT est défavorable sur la rémunération.

Les modalités concrètes de la RTT pourraient, de façon alternative à la démarche retenue ici, être considérées comme davantage liées à l'accord de RTT qu'aux modifications induites par la RTT. Par ailleurs, certains des changements induits par la RTT concernant la visibilité, la variabilité et l'atypicité des horaires ou l'autonomie du salarié peuvent ne pas être indépendants des modalités pratiques d'application de la RTT, ce qui peut affecter les relations statistiques dégagées entre les variables de changements induits par la RTT et la fréquence de satisfaction apportée par la RTT. Aussi, des estimations complémentaires ont été réalisées, traitant les modalités concrètes de la RTT dans la « couche » de variables caractérisant l'accord de RTT (et non plus dans la couche des modifications induites par la RTT). Les résultats de ces estimations subsidiaires - non présentés dans cet article mais disponibles sur simple demande auprès des auteurs - ne diffèrent que marginalement de ceux obtenus par les estimations initiales, ce qui conforte l'analyse.

\section{Les variables d'usage du temps dégagé par la RTT}

La satisfaction serait plus fréquente quand tout ou une partie de ce temps est consacré à la famille, aux activités domestiques, à se reposer ou encore à une autre (ou d'autres) activité(s) de loisirs, mais indépendante du fait de passer plus de temps à des activités de bricolage ou de jardinage (cf. tableau 3).

On a signalé plus haut que la relation statistique dégagée entre la fréquence de satisfaction et l'existence d'un conjoint est sensible à la pré-

15. Pour caractériser les modifications induites par la RTT, un certain nombre de variables synthétiques ont été construites. Les dimensions concernant le caractère régulier ou non des horaires de travail depuis la RTT et la capacité du salarié à connaître ceuxci à l'avance sont appréhendés par une variable qui rend compte à la fois du caractère régulier, alternant ou variable des horaires de travail actuels du salarié et, lorsqu'ils sont variables, du délai avec lequel ils sont connus (juste pour la semaine à venir, entre une semaine et un mois à l'avance, au moins un mois à l'avance). Une autre variable indique la manière dont le délai avec lequel le salarié connaît ses horaires de travail a ou non changé depuis la RTT. 
Tableau 3

Amélioration de la vie quotidienne du fait de la réduction du temps de travail (résultats résumés des estimations logistiques)

\begin{tabular}{|c|c|c|c|c|c|}
\hline & Modèle 1 & Modèle 2 & Modèle 3 & Modèle 4 & Modèle 5 \\
\hline \multicolumn{6}{|c|}{ Variables d'état } \\
\hline $\begin{array}{l}\text { Sexe féminin et enfant à charge de moins de } 12 \text { ans } \\
\text { Âge } \\
\text { Présence d'un conjoint } \\
\text { Diplôme } \\
\text { Catégorie professionnelle } \\
\text { Revenu mensuel du ménage } \\
\text { Habitat urbain } \\
\text { Temps de transport } \\
\text { Effectif de l'entreprise }\end{array}$ & $\begin{array}{l}+ \\
+(-) \\
+ \\
+ \\
+\end{array}$ & $\begin{array}{l}+ \\
(-) \\
+ \\
+ \\
+\end{array}$ & $\begin{array}{l}+ \\
(-)\end{array}$ & $\begin{array}{c}+ \\
+ \\
- \\
+ \\
+(+)\end{array}$ & $\begin{array}{l}+ \\
- \\
\\
+ \\
+(+)\end{array}$ \\
\hline \multicolumn{6}{|c|}{ Variables caractérisant l'accord RTT } \\
\hline $\begin{array}{l}\text { Accord RTT avec aide incitative } \\
\text { Consultation... } \\
\text { - par la direction, l'encadrement intermédiaire } \\
\text { - par un groupe de travail }\end{array}$ & & $\begin{array}{l}+ \\
(+) \\
+\end{array}$ & $\begin{array}{l}+ \\
+ \\
+\end{array}$ & + & + \\
\hline \multicolumn{6}{|c|}{ Modifications induites par la RTT } \\
\hline $\begin{array}{l}\text { Modalités de la RTT } \\
\text { - Journées de travail plus courtes } \\
\text { - Demi-journée ou jour non travaillé chaque semaine, } \\
\text { tous les } 15 \text { jours ou tous les mois } \\
\text { - Jours RTT } \\
\text { - Compte épargne-temps, capital temps, compensation } \\
\text { - Modulation/annualisation } \\
\text { Visibilité et faible variabilité des horaires de travail } \\
\text { Augmentation de la visibilité depuis la RTT } \\
\text { Autonomie dans les horaires de travail } \\
\text { Augmentation de l'autonomie depuis la RTT } \\
\text { Atypicité des horaires de travail : travail entre... } \\
\text { - } 18 \text { et } 20 \text { heures } \\
\text { - } 5 \text { et } 7 \text { heures } \\
\text { - } 20 \text { et } 5 \text { heures } \\
\text { Augmentation de l'atypicité des horaires de travail } \\
\text { La durée effective du travail correspond à celle prévue } \\
\text { dans l'accord } \\
\text { Conditions de travail } \\
\text { - Délais raccourcis } \\
\text { - Objectifs augmentés } \\
\text { Effet défavorable sur la rémunération }\end{array}$ & & & $\begin{array}{l}+ \\
+ \\
+ \\
(+) \\
+ \\
+ \\
+ \\
+ \\
\\
+ \\
- \\
+- \\
+ \\
- \\
(-)\end{array}$ & $\begin{array}{c}+ \\
+ \\
+ \\
(+) \\
+ \\
(+) \\
+ \\
+\end{array}$ & $\begin{array}{c}+ \\
+ \\
(+) \\
+ \\
(+) \\
+ \\
+\end{array}$ \\
\hline \multicolumn{6}{|c|}{ Usages du temps dégagé par la RTT } \\
\hline $\begin{array}{l}\text { Passer plus de temps avec sa famille } \\
\text { Passer plus de temps aux activités domestiques, dont : } \\
\text { - Jardinage ou bricolage } \\
\text { - Autres activités domestiques } \\
\text { Consacrer plus de temps aux loisirs, dont: } \\
\text { - Repos } \\
\text { - Au moins une autre activité de loisir }\end{array}$ & & & & $\begin{array}{l}+ \\
(+) \\
+ \\
+ \\
+\end{array}$ & $\begin{array}{l}+ \\
+ \\
+ \\
+ \\
+\end{array}$ \\
\hline \multicolumn{6}{|c|}{ Questions " subjectives " concernant la période précédant la RTT } \\
\hline $\begin{array}{l}\text { Utilisation du temps : avait réfléchi, } \\
\text { - Mais n'a pas utilisé le temps comme souhaité } \\
\text { - A utilisé le temps comme souhaité } \\
\text { Impression de manquer de temps }\end{array}$ & & & & & $\begin{array}{l}- \\
+ \\
+\end{array}$ \\
\hline
\end{tabular}

Des détails sur le modèle logit sont disponibles en annexe 2.

Ce tableau résume les résultats d'estimations des modèles logistiques (dichotomiques et polytomique) expliquant le sentiment, après une RTT, d'une amélioration, d'une absence de changement ou d'une détérioration de la vie quotidienne. Le nombre de salariés de l'échantillon est de 1618. Les résultats détaillés de ces estimations sont fournis dans les tableaux $A, B$ et $C$ de l'annexe 2.

Les 5 modèles se distinguent par un nombre croissant de variables explicatives retenues. Ainsi, les variables explicatives retenues sont : dans le modèle 1, les seules variables d'état; dans le modèle 2, les variables explicatives du modèle 1 et les variables caractérisant l'accord; dans le modèle 3 , les variables explicatives du modèle 2 et les variables indiquant les modifications induites par la RTT; dans le modèle 4, les variables explicatives du modèle 3 et les variables indiquant les usages du temps dégagé par la RTT; dans le modèle 5 , les variables explicatives du modèle 4 et les variables de réponses aux questions "subjectives " concernant la période précédant l'accord.

Les signes + et - indiquent l'existence d'effets significatifs, respectivement positifs ou négatifs. Le seuil de significativité retenu est $5 \%$, mais les coefficients proches (à un seuil compris entre $5 \%$ et $10 \%$ ) sont cependant fournis entre parenthèses.

Lecture: pour les modèles 3,4 et 5 la fréquence du sentiment d'une amélioration de la vie quotidienne après une RTT serait, toutes choses égales par ailleurs, d'autant plus forte que la durée effective du travail correspond à celle qui était prévue dans l'accord. Source des données de base : enquête RTT et Mode de vie, Dares. 
sence, parmi les variables explicatives, de celles caractérisant l'usage du temps dégagé par la RTT. Cela témoigne d'une relation statistique entre la présence et l'occupation du conjoint et ces usages du temps dégagé. Cette relation concerne plus particulièrement les utilisations du temps « libéré » par la RTT avec sa famille ou à des activités domestiques (cf. tableau 4). Elle est moins significative pour les autres utilisations (repos ou loisirs). Ainsi, très logiquement, l'absence de conjoint s'accompagne d'une faible proportion de salariés utilisant avec leur famille le temps libéré par la RTT. Cette relation partielle entre les deux ensembles de variables explique que la prise en compte des variables caractérisant l'utilisation du temps « libéré » par la RTT affecte les écarts de fréquences de satisfaction des salariés selon la présence ou non d'un conjoint. Il faut donc commenter conjointement ces deux ensembles de variables. Au-delà, il reste cependant à interpréter pourquoi, lorsque l'ensemble de variables explicatives concernant les usages du temps est ajouté à la liste des variables explicatives, la présence d'un conjoint aboutit toujours à une fréquence de satisfaction plus faible des salariés, chacune des occupations possibles du temps dégagé par la RTT augmentant cependant cette fréquence. Une interprétation possible parmi d'autres - de cette interaction serait que la présence d'un conjoint réduit l'autonomie du salarié dans le choix des occupations du temps dégagé par la RTT.
Enfin, en ce qui concerne les questions plus « subjectives » concernant la période précédant la RTT, la satisfaction serait d'autant plus fréquente que le salarié avait réfléchi avant son application à l'usage du temps qui serait dégagé par la RTT. Elle serait également d'autant plus fréquente s'il a pu utiliser ce temps comme il l'envisageait et s'il avait le sentiment de manquer de temps (cf. tableau 3).

Au total, l'approche «toutes choses égales par ailleurs » adoptée permet donc d'approfondir l'analyse en dégageant, de façon plus robuste que les premiers résultats de tris croisés commentés auparavant, les facteurs associés à la satisfaction que la RTT peut apporter aux salariés et de pouvoir mettre en perspective ces résultats avec ceux d'autres approches.

\section{Une comparaison avec les résultats d'autres analyses}

$\mathbf{A}^{\mathrm{r}}$ heure actuelle, très peu d'analyses toutes choses égales par ailleurs » ont été réalisées sur la satisfaction des salariés vis-à-vis d'une RTT déjà mise en œuvre. Les analyses les plus comparables sont celles de Doisneau (2000) et Cette et Diev (2002) réalisées sur une enquête menée en 1999 par l'institut Louis Harris auprès d'environ 500 salariés ayant connu une RTT depuis au moins un an. L'étude de Doisneau (2000) ne procède pas de la même manière, puisqu'elle repose d'abord sur le résultat d'une classification ascendante hiérarchique

Tableau 4

Utilisation du temps « libéré " par la RTT selon l'occupation du conjoint

\begin{tabular}{|c|c|c|c|c|c|c|c|c|c|c|}
\hline \multirow{3}{*}{$\begin{array}{l}\text { Occupation } \\
\text { du conjoint }\end{array}$} & \multicolumn{10}{|c|}{ Cette utilisation consiste à passer plus de temps... } \\
\hline & \multicolumn{2}{|c|}{... avec sa famille } & \multicolumn{2}{|c|}{$\begin{array}{c}\text {... à une activité } \\
\text { domestique de } \\
\text { semi-loisir (jardinage } \\
\text { / bricolage) }\end{array}$} & \multicolumn{2}{|c|}{$\begin{array}{l}\text {... à au moins une } \\
\text { autre activité } \\
\text { domestique que le } \\
\text { jardinage / bricolage }\end{array}$} & \multicolumn{2}{|c|}{... à se reposer } & \multicolumn{2}{|c|}{$\begin{array}{l}\text {... à au moins une } \\
\text { autre activité de } \\
\text { loisir que le repos }\end{array}$} \\
\hline & Non & Oui & Non & Oui & Non & Oui & Non & Oui & Non & Oui \\
\hline Pas de conjoint & 87,3 & 12,7 & 99,7 & 0,3 & 99,3 & 0,7 & 64,0 & 36,0 & 43,8 & 56,2 \\
\hline $\begin{array}{l}\text { Le conjoint ne } \\
\text { travaille pas }\end{array}$ & 33,6 & 66,4 & 79,9 & 20,1 & 88,1 & 11,9 & 64,3 & 35,7 & 45,9 & 54,1 \\
\hline $\begin{array}{l}\text { Le conjoint travaille } \\
\text { à temps partiel }\end{array}$ & 36,6 & 63,4 & 70,5 & 29,5 & 79,8 & 20,2 & 70,5 & 29,5 & 48,1 & 51,9 \\
\hline $\begin{array}{l}\text { Le conjoint travaille } \\
\text { à temps complet }\end{array}$ & 45,2 & 54,8 & 80,8 & 19,2 & 79,6 & 20,4 & 64,0 & 36,0 & 47,5 & 52,5 \\
\hline Ensemble & 50,1 & 49,9 & 82,9 & 17,1 & 84,6 & 15,4 & 64,7 & 35,3 & 46,7 & 53,3 \\
\hline
\end{tabular}

Lecture : parmi les salariés ayant un conjoint travaillant à temps complet, $54,8 \%$ ont déclaré avoir passé plus de temps avec leur famille depuis la RTT. Plusieurs utilisations du temps libéré par la RTT sont possibles.

Source des données de base : enquête RTT et Modes de vie, Dares. 
sur les réponses aux questions concernant la satisfaction des salariés, pour expliquer ensuite, via des estimations logistiques, l'appartenance à chacun des six groupes ainsi constitués par un ensemble de variables. Les résultats obtenus sont, quand la comparaison est possible, tout à fait semblables à ceux présentés ici.

L'approche de Cette et Diev (2002) est plus facilement comparable puisqu'elle consiste aussi, en partie, en une analyse, via des estimations logistiques, de la satisfaction tirée de la RTT par les salariés. Pour autant, le nombre restreint d'observations (environ 500 salariés enquêtés) y a interdit la prise en compte d'un nombre élevé de variables explicatives. Mais il y apparaît, comme ici, que la satisfaction du salarié serait croissante avec sa situation sociale ainsi qu'avec la visibilité et l'autonomie dans les horaires de travail - ce dernier résultat étant très marqué, et décroissante avec l'existence d'un impact défavorable de la RTT sur la rémunération mensuelle. Les résultats présentés dans cet article sont donc cohérents avec ceux de ces précédentes études, mais plus complets compte tenu d'une enquête plus vaste (à la fois en termes de questions posées et du nombre de salariés interrogés).

\section{Des résultats ex post cohérents avec ceux des approches $e x$ ante}

Ces résultats sont aussi en parfaite cohérence avec ceux d'autres études également réalisées toutes choses égales par ailleurs à partir d'enquêtes mais avec une approche ex ante, c'est-à-dire concernant les attentes et représentations des salariés vis-à-vis d'une RTT qu'il leur est demandée d'envisager. Exploitant les réponses à une enquête réalisée en 1995 auprès de 10000 salariés de la chimie, Boulin, Cette et Verger (1998a) aboutissaient ainsi à des enseignements comparables, s'agissant des attentes par rapport à une possible future RTT, que ceux concernant la satisfaction apportée par une RTT effectivement pratiquée au moins une année plus tôt. Ils montraient aussi que les attentes étaient statistiquement en forte relation avec l'appartenance ou la proximité syndicale des salariés, ce que la présente analyse ne peut confirmer concernant la satisfaction, les questions correspondantes n'étant pas posées dans l'enquête RTT et Modes de vie. Pour autant, on peut raisonnablement envisager que l'appartenance ou la proximité syndicale influencent davantage les représentations ex ante vis-à-vis d'une RTT envisagée que la satisfaction appor- tée ex post (et appréciée avec recul) par une RTT effectivement pratiquée.

Cependant, ces résultats peuvent apparaître en décalage avec ceux d'autres enquêtes - de type monographique ou conduites sur des échantillons plus restreints et généralement exploitées par tris croisés - ou avec l'opinion, parfois véhiculée par les médias, selon laquelle la RTT aurait aggravé la situation des salariés en place, notamment des peu qualifiés, et que ses effets sur la vie quotidienne n'auraient donc pas été positifs. Un examen plus attentif permet de s'assurer de la cohérence des principaux résultats issus de ces enquêtes et de celle présentée ici, le décalage apparent provenant sans doute du choix du questionnement et de la grille de lecture utilisée pour l'exploitation.

L'étude réalisée par Pélisse (2002) à partir de six monographies et une cinquantaine d'entretiens met principalement en évidence que la perception des 35 heures par les salariés dépend fortement de la «qualité » des temps « libérés » par la RTT et notamment de la plus ou moins grande maîtrise des temps travaillés et non travaillés. Des salariés parviennent à maîtriser ces temps tandis que, pour d'autres, les contraintes et la flexibilité liées à la modulation sont fortes : «...pour les salariés exprimant autonomie et reconnaissance dans leur travail, (...) l'application des trente cinq heures fait l'objet d'appréciations favorables (...). Les salariés les plus proches des formes d'intégration disqualifiée (incertitude envers l'emploi et insatisfaction dans le travail), ont le plus souvent un discours critique à l'égard des trente cinq heures». Pélisse (2002) indique également que « ... elles (les ouvrières) se plaignent de ne pas avoir été associées aux négociations ou d'avoir été manipulées par les consultants et la direction, mais elles dénoncent surtout le surcroît de flexibilité exigé par les hiérarchies, qui traduit des contraintes temporelles fortes, une polyvalence imposée, destructrice de repères, et des formes de mépris envers leur personne ». Ces enseignements sont cohérents avec les résultats précédents qui montrent que la satisfaction apportée par la RTT est très liée à la fois à la situation sociale du salarié, à la prévisibilité et la maîtrise des horaires de travail et du temps « libéré » par la RTT, et à certaines formes de consultation des salariés concernés. 


\section{Apprécier les effets de la RTT à la fois sur les conditions de travail et sur la vie hors travail}

En revanche, deux éléments permettent d'expliquer l'apparent décalage entre les enseignements de l'enquête RTT et Modes de vie et ceux d'autres sources.

La plupart des enquêtes ont en effet principalement porté sur les transformations intervenues à la suite de la RTT sur les conditions de travail et toutes, y compris l'enquête RTT et Modes de vie, convergent pour mettre en évidence une assez forte dégradation de ces conditions de travail mêlant intensification et surcroît de flexibilité, deux effets qui ne concernent d'ailleurs pas les mêmes catégories. Selon l'enquête RTT et Modes de vie, le motif principal de dégradation des conditions de travail est l'intensification du travail (34\% des cas) et non l'aggravation de la flexibilité (10\%). Mais si, en moyenne, l'intensification du travail est plus souvent mise en avant comme motif principal de dégradation (ce sont proportionnellement les femmes et les hommes cadres ainsi que les hommes de professions intermédiaires qui s'en plaignent le plus), les femmes non qualifiées invoquent beaucoup plus que la moyenne les problèmes de flexibilité et d'imprévisibilité des horaires. Parmi les salariés ayant le sentiment que leur situation quotidienne s'est dégradée, $10 \%$ invoquent la flexibilité comme motif principal, mais cette proportion s'élève à $28 \%$ pour les femmes en horaires alternants ou dont les horaires sont devenus plus variables.

L'enquête RTT et Modes de vie présente la caractéristique d'avoir demandé aux salariés d'exprimer un jugement synthétique des effets de la RTT à la fois sur leurs conditions de travail et sur leur vie «hors travail», ce qui permet sans doute d'expliquer le décalage avec d'autres enquêtes qui n'exigeaient pas cette synthèse complexe. Ainsi, dans les réponses à l'enquête RTT et Modes de vie, on passe d'une proportion assez faible de salariés considérant que leurs conditions de travail se sont améliorées $(26 \%)$ à une forte majorité (59\%) de salariés considérant que, dans l'ensemble, l'effet de la RTT a plutôt été dans le sens d'une amélioration de la vie quotidienne, tout ayant été bien pesé. Les réponses à la question ouverte permettent d'ailleurs assez souvent de rencontrer des constructions comme : «ça a dégradé la qualité de travail, par contre j'ai une amélioration de ma qualité de vie personnelle ». L'exploitation des réponses à cette question ouverte met en évi- dence que le sentiment d'amélioration est quasi exclusivement rapporté au domaine du «hors travail », cependant que celui de dégradation mentionne le seul domaine du travail (16).

\section{Vers une meilleure conciliation entre vie professionnelle et vie familiale ?}

La grille de lecture utilisée pour exploiter l'enquête a aussi fait une large place, au-delà des variables d'état (âge, sexe, situation sociale, etc.) à la prise en compte conjointe du sexe et de la présence d'enfant(s) et à celle des attentes subjectives des personnes interrogées, démarche qui avait été peu empruntée par les analyses exploitant d'autres enquêtes et qui s'est révélée riche de sens.

Elle a en effet permis de caractériser un groupe de personnes ayant de fortes attentes vis-à-vis de la RTT (des salariés travaillant à temps complet ayant de jeunes enfants et donc souvent confrontés à un sentiment de manque de temps, plus particulièrement les femmes dans cette situation, étant donnée la répartition actuelle des rôles dans notre société). Ainsi, les personnes déclarant le plus fréquemment qu'elles manquaient de temps avant la RTT sont les salariés avec au moins un enfant de moins de 12 ans, les femmes (pour $57 \%$ de celles dans cette situation) plus encore que les hommes $(40 \%)$. Ce manque de temps était principalement référé à la famille : $63 \%$ des personnes qui avaient réfléchi à ce qu'elles feraient d'un surcroît de temps et $89 \%$ des femmes avec enfants de moins de 12 ans dans la même situation déclaraient souhaiter consacrer ce surcroît de temps à leur famille. Et l'un des résultats importants de l'analyse présentée ici est bien de montrer que, toutes choses égales par ailleurs, les femmes avec au moins un enfant de moins de 12 ans seraient plus souvent satisfaites de la RTT que les autres salariés.

Un tel résultat vient compléter une lecture principalement centrée sur les catégories professionnelles et amène à en affiner les résultats. L'analyse par tris croisés avait mis en évidence que le jugement des salariés sur les effets de la RTT est très lié à la catégorie professionnelle, ou plus exactement au croisement de la catégorie professionnelle et du sexe : le niveau de satisfaction des hommes non qualifiés est proche $(57 \%)$ de celui de la moyenne $(59 \%)$,

16. Cf. Defalvard et Méda (2003). 
les femmes ouvrières ou employées non qualifiées se déclarant beaucoup moins satisfaites que la moyenne $(40 \%)$. Une analyse plus approfondie montre que ces femmes de l'échantillon ont moins d'enfants que les femmes cadres ou de professions intermédiaires, ce fait rejoignant les résultats d'autres enquêtes montrant que les femmes non qualifiées sont moins nombreuses que les autres à se maintenir sur le marché du travail lorsqu'elles ont des enfants. Les femmes ouvrières ou employées non qualifiées de l'échantillon, lorsqu'elles ont des enfants de moins de 12 ans, sont proportionnellement plus satisfaites que les autres femmes non qualifiées sans enfant.

Ainsi, prendre simultanément en considération le sexe et la présence d'enfants aboutit à des résultats différents d'une approche se référant aux seules catégories professionnelles. L'analyse demanderait certes à être beaucoup plus poussée, mais l'un des enseignements obtenus est que la conciliation entre vie professionnelle et vie familiale semble avoir été particulièrement améliorée pour les femmes avec enfant(s) de moins de 12 ans. Cette dimension de meilleure conciliation entre vie professionnelle et vie familiale n'avait pas été très présente lors de l'élaboration des dispositifs législatifs et réglementaires de RTT ou lors des négociations de RTT. L'un des mérites de l'enquête RTT et Modes de vie est sans doute de faire apparaître que cette dimension est essentielle. Pour les femmes ayant la charge de jeune(s) enfant(s), une meilleure conciliation peut signifier une meilleure insertion dans la vie professionnelle, de moindres renoncements à l'activité, notamment pour les moins qualifiées, et donc des taux d'activité féminins plus élevés.

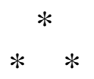

L'analyse multivariée réalisée sur les réponses à l'enquête RTT et Modes de vie auprès de salariés ayant connu une RTT depuis au moins une année dans le cadre des dispositifs « Robien » et «Aubry 1 » permet donc d'apprécier les liens, toutes choses égales par ailleurs, entre de nombreuses variables regroupées en cinq catégories et la satisfaction des salariés vis-à-vis de la RTT.

Certaines caractéristiques semblent toujours exercer une influence sur le jugement des salariés vis-à-vis de la RTT et concourir au renforcement de la satisfaction. Ainsi, les femmes ayant à charge un enfant de moins de 12 ans sembleraient, toutes choses égales par ailleurs, trouver plus de satisfaction à la RTT que les autres salariés interrogés. Par ailleurs, les facteurs influençant significativement la satisfaction seraient notamment la situation sociale du salarié (appréciée conjointement par son diplôme, sa catégorie professionnelle et le revenu du ménage), la visibilité des horaires de travail et son amélioration, l'autonomie dans les horaires de travail, le fait que la durée effective correspond bien à celle qui était prévue dans l'accord, l'impact de la RTT sur la rémunération et les usages qui sont faits du temps dégagé par la RTT. Parmi ces usages, on remarque principalement l'augmentation du temps passé avec la famille (conjoint et enfants) et du temps passé à se reposer ou à exercer d'autres activités domestiques, de semi-loisir ou de loisir. Le fait que le salarié ait eu, avant la RTT, le sentiment de manquer de temps, qu'il ait réfléchi à l'usage du temps potentiellement dégagé par la RTT, et que cet usage se soit traduit concrètement dans la réalité influencerait également sa satisfaction. Inversement, la variabilité des horaires, l'atypicité des horaires de manière générale et son augmentation, la hausse des objectifs et l'impact défavorable de la RTT sur la rémunération influenceraient négativement cette satisfaction.

Outre ces variables fortement influentes, avoir un habitat urbain influencerait aussi positivement la satisfaction. D'autre part, la satisfaction serait plus rare quand le salarié a un conjoint que lorsqu'il n'en a pas. Enfin, la satisfaction du salarié serait plus fréquente dès lors que l'accord s'accompagne d'une aide incitative et la consultation (via la direction de l'entreprise, l'encadrement intermédiaire ou autres groupes de travail) influencerait également de manière positive le jugement du salarié, mais dans une moindre mesure.

Pour autant, cette étude et ses conclusions restent partielles pour des raisons quantitatives (le nombre d'individus interrogés est encore restreint) et qualitatives (les accords de type «Aubry 2 » ne sont pas pris en compte, et bon nombre de variables explicatives sont finalement assez frustes). Les résultats présentés apparaissent toutefois assez robustes. Ils permettent de nourrir la réflexion sur les déterminants du jugement des salariés vis-à-vis de la RTT, ce jugement jouant lui-même un rôle prépondérant dans les conditions de réussite économique d'un tel processus. Mais ils demandent à être confortés par des analyses ultérieures. 


\section{BIBLIOGRAPHIE}

Boulin J.-Y., Cette G. et Verger D. (1998a), «Les salariés de la chimie face à la réduction du temps de travail », document de travail, $n^{\circ} 9801$, Crest.

Boulin J.-Y., Cette G. et Verger D. (1998b), «Les arbitrages entre temps libre et salaires: enseignements d'une enquête réalisée auprès des salariés de la chimie », Travail et Emploi, $\mathrm{n}^{\circ} 77$, 4/98.

Boulin J.-Y., Lallement M., Lefèvre G. et Silvéra R. (1998), « Temps de travail et mode de vie: quelques résultats d'une enquête empirique », Futuribles, n²37, décembre.

Cette G. et Diev P. (2002), «La réduction du temps de travail : qu'en pensent et qu'en font les salariés qui la vivent?», mimeo. Une partie de cette étude a été publiée dans Futuribles $n^{\circ} 285$, avril 2003, sous le titre «L'impact de la réduction du temps de travail ».

CFDT (1999), « 10000 salariés s'expriment sur la réduction du temps de travail un an après un accord d'entreprise », Le temps de travail en question, $\mathrm{n}^{\circ} 6$, octobre.

CFDT (2001), Le Travail en questions, Éditions Syros.

Commissariat général du Plan (2001), Réduction du temps de travail: les enseignements de l'observation, rapport de la Commission présidée par Henri Rouilleaut, La documentation Française, juillet.

Defalvard H. et Méda D. (2003), «Les mondes vécus des trente cinq heures », Recherches et prévisions, $\mathrm{n}^{\circ} 74$, décembre.

Doisneau L. (2000), « Les accords Robien un an après: l'expérience des salariés », Travail et Emploi, $\mathrm{n}^{\circ} 83$, juillet.

Économie Internationale (2000), « Réduction du temps de travail et Emploi », $n^{\circ} 83,3^{\mathrm{e}}$ trimestre.
Estrade M.-A. et Méda D. (2002), « Principaux résultats de l'enquête RTT - Modes de vie », Document d'études de la Dares, $\mathrm{n}^{\circ}$ 56, mai.

Estrade M.-A., Méda D. et Orain R. (2001), «Les effets de la RTT sur les modes de vie : qu'en pensent les salariés un an après ? ", Premières informations, $\mathrm{n}^{\circ}$ 21.1, Dares, ministère des Affaires sociales, du Travail et de la Solidarité, mai.

Estrade M.-A. et Ulrich V. (2002), « La réorganisation des temps travaillés et les trente cinq heures : un renforcement de la segmentation du marché du travail », Travail et Emploi, $\mathrm{n}^{\circ}$ 92, octobre.

Lollivier S., Marpsat M. et Verger D. (1996), «L'économétrie et l'étude des comportements : présentation et mise en œuvre de modèle de régression qualitatifs », Ensae, Série des Documents de Travail, Méthodologie Statistique.

Méda D. et Delteil V. (2002), « Les cadres face à la réduction du temps de travail : toujours loin des 35 heures, mais plus satisfaits en termes de temps dégagé », Revue française de gestion, septembre.

Méda D. et Orain R. (2002), «Transformations du travail et du « hors-travail» : le jugement des salariés sur la RTT », Travail et Emploi, n 90, avril.

MES (1999), La réduction du temps de travail : les enseignements des accords (été 1998, été 1999), La documentation Française, septembre.

Pélisse J. (2002), «À la recherche du temps gagné : les trente cinq heures entre perceptions, régulations et intégrations professionnelles », Travail et Emploi, ${ }^{\circ}$ 90, avril.

Romanowski S. (2002), «RTT: le travail en questions », in «Le devenir des trente cinq heures », Revue de la CFDT, avril-mai.

Travail et Emploi (2000a), « Premiers regards sur les 35 heures », $n^{\circ} 82$, avril.

Travail et Emploi (2000b), « Premiers effets des 35 heures $», n^{\circ} 83$, juillet. 


\section{L'ENQUÊTE RTT ET MODES DE VIE}

On présente successivement l'enquête et les variables qui en sont issues et qui interviennent dans les estimations logistiques réalisées.

\section{Une enquête réalisée directement auprès des salariés}

Conçue par une équipe de la Dares (Mission Animation de la Recherche et Mission Analyse Économique) assistée d'un conseil scientifique, l'enquête RTT et Mode de vie est la seconde enquête quantitative auprès de salariés ayant connu un processus collectif de réduction de la durée du travail. La précédente avait eu lieu auprès de 526 salariés, travaillant dans des entreprises ayant réduit la durée du travail dans le cadre d'un dispositif "Robien ". Un contact préalable avait été pris avec un échantillon d'entreprises afin d'avoir accès à leur fichier de salariés. Cette première enquête s'est toutefois heurtée à un problème majeur : près des deux tiers des entreprises avaient soit refusé l'accès à leur fichier, soit n'avaient pu être contactées à temps. Les raisons de ce refus ou de ce retard n'ont pas toujours été explicites, mais les observations des enquêteurs ont fait ressortir que de nombreux chefs d'entreprise n'avaient pas souhaité rouvrir le débat sur les 35 heures. Une telle situation constitue un biais notable dans l'échantillon, dans la mesure où, sans doute, seuls les salariés d'entreprises où la RTT s'était bien déroulée ont été interrogés.

Pour l'enquête RTT et Modes de Vie, la Dares a donc fait le choix de ne pas passer par les employeurs, mais plutôt de contacter directement les salariés, par l'intermédiaire des déclarations annuelles de données sociales (DADS). Ce choix impliquait cependant des traitements relativement lourds.

Au début de l'année 2000, la Dares a constitué un fichier de l'ensemble des Siren (12 700) correspondant à des entreprises ayant réduit leur durée du travail avant novembre 1999. Les dispositifs législatifs dans le cadre desquels la RTT a eu lieu sont les conventions «Robien » (offensives et défensives) et les accords "Aubry 1 " (offensifs et défensifs). Ce fichier de Siren a été transmis à l'Insee au début de l'année 2000 , afin qu'il récupère dans les DADS, les adresses et informations concernant l'ensemble des salariés de ces entreprises. Le fichier issu de ce dernier traitement était " brut de collecte ", non redressé. La Dares a donc mis au point les procédures de redressement afin de mettre en cohérence ces différentes informations.

Une deuxième opération a conduit la Dares à retravaille les informations dont elle disposait à propos des accords et conventions, afin de mieux cerner d'une part le périmètre de la RTT mise en œuvre dans chaque entreprise, et d'autre part de déterminer le(s) horaire(s) collectif(s) appliqué(s). La concordance de ces informations a alors été comparée au niveau de chaque établissement, ce qui a amené, de fait, à écarter de nombreuses entreprises pour lesquelles les incohérences étaient trop importantes.
Une nouvelle réduction de l'échantillon a été par la suite effectuée, afin de ne conserver que les individus les plus susceptibles de rentrer dans le champ de l'enquête, en supprimant par exemple les VRP et cadres dirigeants généralement non concernés par la RTT. Cette opération a conduit à ne conserver que 80000 salariés. Afin de limiter le biais, un calage sur le nombre de salariés ayant effectivement réduit leur durée du travail à la fin du $3^{\mathrm{e}}$ trimestre 1999, par loi et taille d'entreprise (grâce aux données issues de l'enquête Acemo), a permis de déterminer les poids d'inclusion dans le fichier.

Cette base de 80000 salariés a permis de définir les quotas, à savoir les variables de stratification de l'enquête. Cette procédure a aussi permis de s'assurer que la structure des salariés interrogés respecterait les informations paraissant ex ante les plus importantes pour la compréhension de l'impact de la RTT. Les variables ayant servi à déterminer lesdits quotas sont le sexe, l'âge, la distinction cadre/non-cadre, le secteur d'activité et une variable sur le type de commune. Certaines catégories ont été regroupées car elles concernaient un trop faible nombre de personnes. 73 catégories ont ainsi été définies, un nombre fixé à l'avance de questionnaires devant être rempli dans chacune d'entre elles.

Un échantillon de 17600 adresses a été sélectionné. Afin de limiter le coût de déplacement des enquêteurs, certaines communes de résidence des salariés ont été éliminées.

Le choix de cette méthode de sondage ne va pas de soi. En effet, il y a un risque important que les répondants soient « volontaires » ou du moins soient les salariés les plus faciles à contacter. Afin de pallier, dans une certaine mesure, le risque de biais introduit par la méthode, et sur conseil du comité de label, le fichier des adresses a été livré en trois fois (8 000 adresses les deux premières fois, puis une troisième livraison de 1000 adresses). Pour le premier fichier livré, on a ainsi pu s'assurer que toutes les adresses avaient été exploitées, à différents moments de la journée et de la semaine. Les taux de hors-champ calculés sur ce premier fichier ont servi pour la détermination de la pondération.

Chacun de ces fichiers d'adresses a fait l'objet d'une recherche téléphonique : entre $55 \%$ et $60 \%$ de salariés ont été retrouvés. Une lettre présentant l'enquête a été envoyée. Le salarié a été contacté par téléphone pour déterminer son appartenance au champ de l'enquête et lui faire accepter le principe d'une interview à domicile.

Les taux de hors-champ ont été relativement élevés, principalement pour deux raisons : soit la personne ne travaillait plus dans la même entreprise, soit elle avait l'impression de ne pas avoir réduit sa durée de travail. À l'issue de cette phase téléphonique, 1618 salariés ont ainsi accepté de recevoir un enquêteur en face-à-face, dans la plupart des cas à leur domicile.

Les enquêtes sur le terrain se sont déroulées de novembre 2000 à février 2001. La durée de l'interview a été de 50 minutes en moyenne, mais cette durée a été beaucoup plus longue pour les ménages avec enfants. 
Un questionnaire pour évaluer l'ensemble des effets de la RTT...

Le questionnaire, préparé par la Dares, avait pour but de cerner l'ensemble des effets de la RTT sur les modes de vie des salariés, tant au travail qu'en dehors de la sphère du travail (vie familiale, activités domestiques, loisirs, partage des tâches et usages du temps, etc.). À cet effet, le questionnaire aborde différents thèmes: l'accord de $\mathrm{RTT}$, les modalités de réduction du temps de travail, la réduction effective, l'organisation des horaires et du nombre de jours de travail, les conditions de travail, les congés, la vie familiale, les tâches domestiques, les loisirs, les revenus et des questions sur les attentes des salariés et le bilan global. L'objectif de l'enquête étant de connaître les effets "structurels ", pour les salariés, du processus de RTT, il a été choisi de n'interroger que des salariés d'entreprises ayant mis en œuvre une RTT au moins un an avant l'enquête. Cela permet, par exemple, aux salariés qui travaillent selon des horaires modulés sur l'année, d'avoir ainsi connu l'ensemble des périodes hautes et basses de durée de travail.

Par définition, aucun salarié de l'échantillon n'est concerné par le dispositif "Aubry 2 ", établi par la loi du 19 janvier 2000. L'enquête s'est restreinte aux salariés ayant connu l'ensemble du processus de RTT, c'est-àdire présents dans l'établissement avant l'accord, toujours présents dans l'établissement au moment de l'enquête, et ayant réduit, même faiblement, leur durée de travail. D'autre part, seuls les salariés à temps complet sont concernés par cette enquête.

Les premiers résultats, par tris croisés, ont été publiés dans Estrade, Méda, Orain (2001). Cette enquête a auss servi de support à une première analyse, également par tris croisés, de l'appréciation des salariés bénéficiant d'une RTT dans Méda et Orain (2002).

\section{... pour les salariés passés à 35 heures depuis au moins un an}

La structure des salariés passés à 35 heures depuis au moins un an a pu changer depuis l'enquête, mais cela ne pénalise en rien les résultats d'analyses basées, comme ici, sur des estimations logistiques. En effet, ce qui importe dans de telles analyses est que chaque catégorie de la population à représenter (ici les salariés ayant connu une RTT depuis au moins un an) soit effectivement représentée dans l'échantillon, et non que la proportion de chacune de ces catégories soit identique dans l'échantillon à celui observé dans la population à représenter.

Dans les classifications qui suivent, sont considérés comme ouvriers et employés qualifiés les ouvriers qualifiés de types industriel et artisanal, les chauffeurs, les ouvriers qualifiés de la manutention, du magasinage et du transport, les policiers et militaires, les employés administratifs d'entreprise. Sont considérés comme ouvriers et employés non qualifiés les ouvriers non qualifiés de types industriel et artisanal, les ouvriers agricoles, les employés de commerce et les personnels de service direct aux employeurs.

Pour qualifier le « type d'habitat » du salarié, on a retenu une typologie inspirée de la nouvelle nomenclature spatiale élaborée par l'Insee, à savoir le zonage en aires urbaines (ZAU). II est donc utile de connaitre les définitions principales des zones en question. Le pôle urbain est une unité urbaine qui offre au moins 5000 emplois sur son territoire, sous réserve qu'elle ne soit pas sous la dépendance directe d'un pôle urbain plus important. L'aire urbaine est constituée d'un pôle urbain auquel on a agrégé les communes rurales ou unités urbaines qui y envoient au moins $40 \%$ de leurs actifs ayant un emploi (un processus itératif permet d'agréger, dans un second temps, les autres communes rurales ou unités urbaines envoyant $40 \%$ de leur population active vers cet ensemble en cours de construction). La couronne périurbaine est constituée, dans chaque aire urbaine, des communes rurales ou unités urbaines n'appartenant pas au pôle urbain.

Les communes multipolarisées sont les communes rurales ou petites unités urbaines envoyant $40 \%$ de leurs actifs ayant un emploi vers plusieurs aires urbaines, sans atteindre ce seuil avec une seule d'entre elles, et qui forment avec elles un ensemble connexe. Enfin, l'espace à dominante rurale est l'ensemble des communes ou petites unités n'appartenant pas à l'espace à dominante urbaine.

Le questionnaire de l'enquête, trop « lourd " pour être ici reproduit (56 pages), peut être demandé aux auteurs.

\section{Expliquer la variable de satisfaction}

Les variables utilisées dans l'analyse proviennent toutes des réponses à l'enquête RTT et Modes de vie.

La variable de satisfaction, qui est la variable expliquée intervenant dans les estimations logistiques, correspond aux réponses à la question (10.5.1 du questionnaire) :

"Globalement, diriez-vous que les effets de la RTT sur votre vie quotidienne, aussi bien au travail qu'en dehors du travail, ont été :

\section{Plutôt dans le sens d'une amélioration ?}

\section{Plutôt dans le sens d'une dégradation?}

\section{Cela n'a rien changé. »}

La formulation de cette question est volontairement générale afin de pouvoir intégrer les diverses dimensions de la satisfaction tirée avec recul par les salariés du processus de RTT qu'ils ont connu. Les réponses aux trois modalités possibles se répartissent comme indiqué dans le tableau 1 de l'article.

Parmi les très nombreuses questions posées par l'enquête, seules certaines (en nombre déjà important) ont été retenues pour constituer les variables explicatives de l'analyse. Le choix de ces variables et de leurs modalités a été effectué sur la base de standards usuels (pour les variables d'état par exemple), des enseignements de précédentes analyses (cf. bibliographie), de la qualité des résultats obtenus (pour choisir parfois entre des variables redondantes, par exemple), et d'effectifs en nombre suffisant dans chaque modalité.

Le tableau suivant fournit un dénombrement des diverses modalités de réponses aux questions constituant les variables explicatives de l'analyse. 
Dénombrement des modalités des variables explicatives de l'analyse

\begin{tabular}{|c|c|c|c|}
\hline Variables & Modalités & Effectifs & Proportions (en \%) \\
\hline \multicolumn{4}{|c|}{ Variables d'état } \\
\hline Sexe et enfant & $\begin{array}{l}\text { Femme avec enfant de moins de } 12 \text { ans } \\
\text { Autre femme } \\
\text { Homme avec enfant de moins de } 12 \text { ans } \\
\text { Autre homme }\end{array}$ & $\begin{array}{l}151 \\
377 \\
480 \\
610\end{array}$ & $\begin{array}{r}9,33 \\
23,30 \\
29,67 \\
37,70\end{array}$ \\
\hline Âge & $\begin{array}{l}15 \text { (borne inférieure) à } 29 \text { ans } \\
30 \text { à } 39 \text { ans } \\
40 \text { à } 49 \text { ans } \\
50 \text { ans et plus }\end{array}$ & $\begin{array}{l}189 \\
543 \\
564 \\
322\end{array}$ & $\begin{array}{l}11,68 \\
33,56 \\
34,86 \\
19,90\end{array}$ \\
\hline Diplôme & $\begin{array}{l}\text { Aucun diplôme } \\
\text { Enseignement court } \\
\text { Niveau secondaire } \\
\text { Enseignement supérieur }\end{array}$ & $\begin{array}{r}91 \\
850 \\
256 \\
421\end{array}$ & $\begin{array}{r}5,62 \\
52,53 \\
15,82 \\
26,02\end{array}$ \\
\hline Catégorie professionnelle & $\begin{array}{l}\text { Employé ou ouvrier non qualifié } \\
\text { Employé ou ouvrier qualifié } \\
\text { Profession Intermédiaire } \\
\text { Cadre }\end{array}$ & $\begin{array}{l}214 \\
747 \\
411 \\
246\end{array}$ & $\begin{array}{l}13,23 \\
46,17 \\
25,40 \\
15,20 \\
\end{array}$ \\
\hline Revenu mensuel du ménage & $\begin{array}{l}\text { Moins de } 10000 \text { francs } \\
\text { De } 10001 \text { à } 15000 \text { francs } \\
\text { De } 15001 \text { à } 20000 \text { francs } \\
\text { De } 20001 \text { à } 25000 \text { francs } \\
\text { Plus de } 25000 \text { francs }\end{array}$ & $\begin{array}{l}326 \\
483 \\
430 \\
193 \\
186\end{array}$ & $\begin{array}{l}20,15 \\
29,85 \\
26,58 \\
11,93 \\
11,50\end{array}$ \\
\hline Occupation du conjoint & $\begin{array}{l}\text { Pas de conjoint } \\
\text { Le conjoint ne travaille pas } \\
\text { Le conjoint travaille à temps partiel } \\
\text { Le conjoint travaille à temps complet et connaît une } \\
\text { RTT avec modulation } \\
\text { Le conjoint travaille à temps complet et connaît une } \\
\text { RTT sans modulation } \\
\text { Le conjoint travaille à temps complet sans RTT }\end{array}$ & $\begin{array}{l}292 \\
244 \\
183 \\
144 \\
300 \\
455\end{array}$ & $\begin{array}{r}18,05 \\
15,08 \\
11,31 \\
8,90 \\
18,54 \\
28,12\end{array}$ \\
\hline Type d'habitat & $\begin{array}{l}\text { Rural } \\
\text { Multi-polarisé } \\
\text { Périurbain } \\
\text { Pôle urbain hors Paris et région parisienne } \\
\text { Paris et région parisienne }\end{array}$ & $\begin{array}{l}260 \\
103 \\
365 \\
704 \\
186\end{array}$ & $\begin{array}{r}16,07 \\
6,37 \\
22,56 \\
43,51 \\
11,50\end{array}$ \\
\hline Temps de transport & $\begin{array}{l}\text { Moins de } 15 \text { minutes } \\
\text { Entre } 15 \text { minutes et une demi-heure } \\
\text { Plus d'une demi-heure }\end{array}$ & $\begin{array}{l}650 \\
720 \\
248\end{array}$ & $\begin{array}{l}40,17 \\
44,50 \\
15,33\end{array}$ \\
\hline Effectif de l'entreprise & $\begin{array}{l}\text { Moins de } 51 \text { salariés } \\
\text { De } 51 \text { à } 150 \text { salariés } \\
\text { De } 151 \text { à } 550 \text { salariés } \\
\text { De } 551 \text { à } 2000 \text { salariés } \\
\text { Plus de } 2000 \text { salariés }\end{array}$ & $\begin{array}{l}192 \\
259 \\
358 \\
407 \\
402\end{array}$ & $\begin{array}{l}11,87 \\
16,01 \\
22,13 \\
22,15 \\
24,85\end{array}$ \\
\hline \multicolumn{4}{|c|}{ Variables caractérisant l'accord RTT } \\
\hline Type d'accord & $\begin{array}{l}\text { RTT sans aide incitative } \\
\text { Robien défensif } \\
\text { Robien offensif } \\
\text { Aubry I défensif } \\
\text { Aubry I offensif }\end{array}$ & $\begin{array}{l}348 \\
174 \\
263 \\
121 \\
712 \\
\end{array}$ & $\begin{array}{r}21,51 \\
10,75 \\
16,25 \\
7,48 \\
44,00 \\
\end{array}$ \\
\hline $\begin{array}{l}\text { Consultation : } \\
\text { - Par la direction ou l'encadrement } \\
\text { intermédiaire }\end{array}$ & $\begin{array}{l}\text { Non } \\
\text { Oui }\end{array}$ & $\begin{array}{l}781 \\
837\end{array}$ & $\begin{array}{l}48,27 \\
51,73\end{array}$ \\
\hline $\begin{array}{l}\text { - Par les syndicats ou les élus du per- } \\
\text { sonnel }\end{array}$ & $\begin{array}{l}\text { Non } \\
\text { Oui }\end{array}$ & $\begin{array}{l}790 \\
828\end{array}$ & $\begin{array}{l}48,83 \\
51,17\end{array}$ \\
\hline - Par un ou des groupe(s) de travail & $\begin{array}{l}\text { Non } \\
\text { Oui }\end{array}$ & $\begin{array}{r}1346 \\
272\end{array}$ & $\begin{array}{l}83,19 \\
16,81\end{array}$ \\
\hline
\end{tabular}


Dénombrement des modalités des variables explicatives de l'analyse (suite)

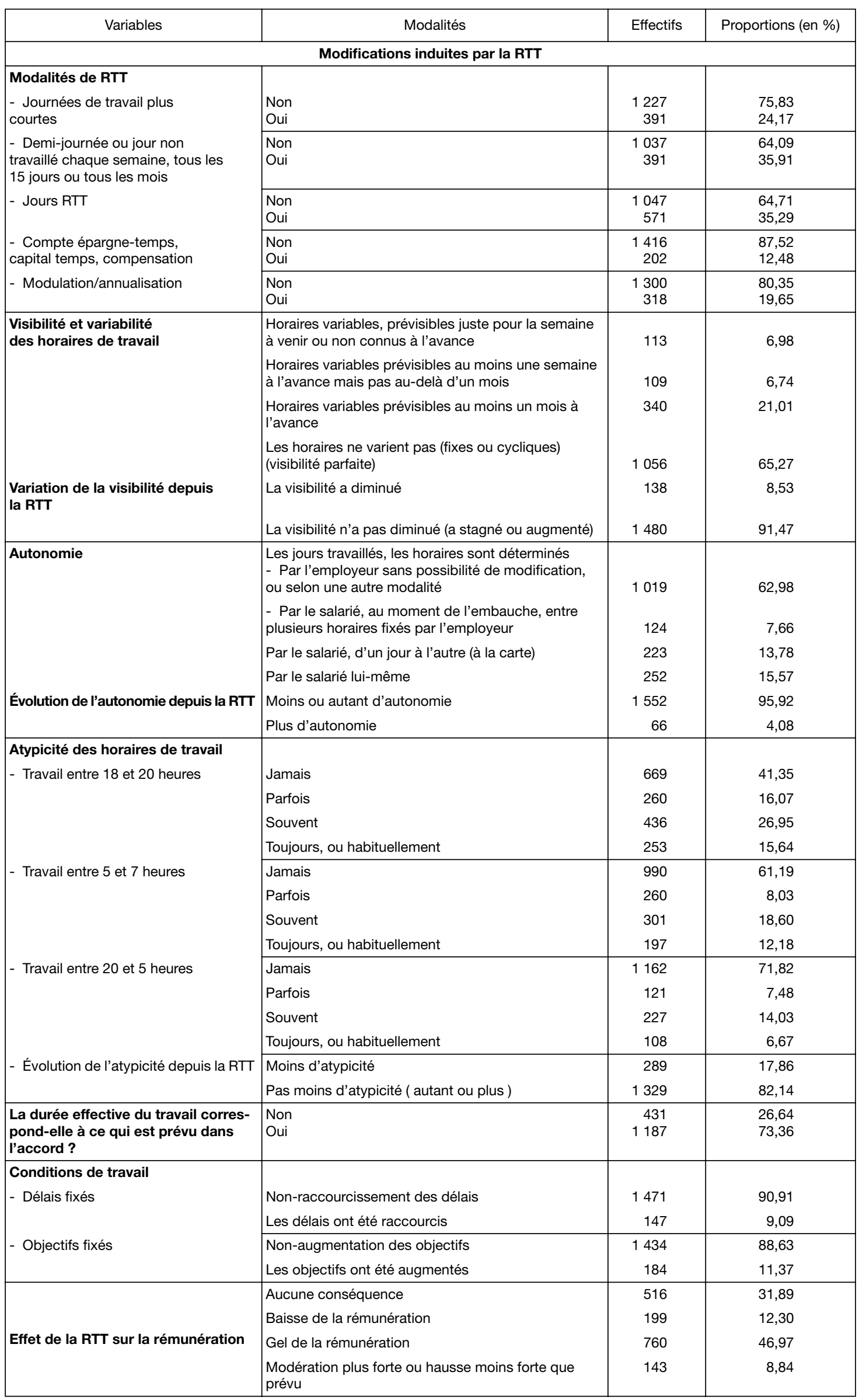


Dénombrement des modalités des variables explicatives de l'analyse (fin)

\begin{tabular}{|c|c|c|c|}
\hline Variables & Modalités & Effectifs & Proportions (en \%) \\
\hline \multicolumn{4}{|c|}{ Usages du temps dégagé par la RTT } \\
\hline Passer plus de temps avec sa famille & $\begin{array}{l}\text { Non } \\
\text { Oui }\end{array}$ & $\begin{array}{l}810 \\
808\end{array}$ & $\begin{array}{l}50,06 \\
49,94\end{array}$ \\
\hline $\begin{array}{l}\text { Activités domestiques, dont: } \\
\text { - Faire plus de jardinage ou de brico- } \\
\text { lage }\end{array}$ & $\begin{array}{l}\text { Non } \\
\text { Oui }\end{array}$ & $\begin{array}{r}1341 \\
277\end{array}$ & $\begin{array}{l}82,88 \\
17,12\end{array}$ \\
\hline $\begin{array}{l}\text { - Passer plus de temps à au moins } \\
\text { une autre activité domestique }\end{array}$ & $\begin{array}{l}\text { Non } \\
\text { Oui }\end{array}$ & $\begin{array}{r}1368 \\
250\end{array}$ & $\begin{array}{l}84,55 \\
15,45\end{array}$ \\
\hline $\begin{array}{l}\text { Loisirs } \\
\text { - Passer plus de temps à se reposer }\end{array}$ & $\begin{array}{l}\text { Non } \\
\text { Oui }\end{array}$ & $\begin{array}{r}1048 \\
570\end{array}$ & $\begin{array}{l}66,77 \\
35,23\end{array}$ \\
\hline $\begin{array}{l}\text { - Passer davantage de temps à au } \\
\text { moins une activité de loisir autre que le } \\
\text { repos }\end{array}$ & $\begin{array}{l}\text { Non } \\
\text { Oui }\end{array}$ & $\begin{array}{l}755 \\
863\end{array}$ & $\begin{array}{l}46,66 \\
53,34\end{array}$ \\
\hline \multicolumn{4}{|c|}{ Questions " subjectives " concernant la période précédant la RTT } \\
\hline Réflexion sur l'utilisation du temps & $\begin{array}{l}\text { N'avait pas réfléchi } \\
\text { Avait réfléchi mais n'a pas utilisé le temps comme } \\
\text { souhaité } \\
\text { Avait réfléchi et a utilisé le temps comme souhaité }\end{array}$ & $\begin{array}{r}1046 \\
173 \\
399\end{array}$ & $\begin{array}{l}64,65 \\
10,69 \\
24,66\end{array}$ \\
\hline Impression de manquer de temps & \begin{tabular}{|l} 
Jamais \\
Rarement \\
Parfois \\
Souvent \\
Toujours
\end{tabular} & $\begin{array}{l}407 \\
158 \\
415 \\
339 \\
279\end{array}$ & $\begin{array}{r}25,15 \\
9,77 \\
25,65 \\
22,19 \\
17,24\end{array}$ \\
\hline
\end{tabular}

Source des données de base : enquête RTT et Modes de vie, Dares. 


\section{DES ESTIMATIONS LOGISTIQUES QUALITATIVES}

On présente successivement le principe des estimations logistiques (1), puis les résultats détaillés des estimations en les commentant.

\section{Des modèles à variables qualitatives}

L'économétrie des variables qualitatives, et notamment les modèles Logit ou Probit, permettent d'isoler l'effet propre d'un facteur (le sexe du salarié par exemple) sur une caractéristique (ici la satisfaction vis-à-vis de la $\mathrm{RTT}$ ). C'est la raison pour laquelle les résultats issus de tels modèles sont à commenter caeteris paribus (« toutes choses égales par ailleurs »).

Deux types de modèles à variables qualitatives sont utilisés dans cette étude: un modèle logit simple (cf. tableaux $\mathrm{A}$ et $\mathrm{B}$ ) et un modèle logit polytomique ordonné (cf. tableau C). Le modèle polytomique ordonné est utilisé lorsque la variable à expliquer peut prendre plus de deux modalités (le modèle est alors polytomique), si ces modalités sont ordonnées de façon explicite. Dans cet article, la variable expliquée peut prendre trois modalités : amélioration, absence de changement ou dégradation de la vie quotidienne (en l'occurrence, le modèle polytomique correspondant sera dit logit trichotomique). Le modèle logit simple est un cas particulier du modèle polytomique, la variable à expliquer n'ayant que deux modalités possibles. Ici il s'agit, par exemple, de distinguer les salariés ayant connu une amélioration de leur vie quotidienne de ceux qui ont connu ou une absence de changement ou une dégradation, ou de distinguer les salariés ayant connu une dégradation de ceux ayant connu soit une amélioration soit une absence de changement. Dans l'approche polytomique, on cherche à confirmer les résultats obtenus par les deux autres types de logit simple en considérant les trois modalités de la variable expliquée comme des degrés ordonnés d'amélioration de la vie quotidienne (du sentiment de dégradation au sentiment d'amélioration, en passant par le sentiment de statu quo). Il est alors important de savoir s'il est de bon alo de considérer ce modèle logistique trichotomique comme "ordonné », ou s'il est préférable de l'appréhender comme un modèle logit multinomial non ordonné. Le test de l'hypothèse d'égalité des pentes («Score test for the proportional odds assumption») donne une valeur, qui grâce à un test du Chi-deux permet de répondre à cette question. Si la probabilité que cette statistique soit supérieure à la valeur du Chi-deux est inférieure à $5 \%$, on peut considérer que le modèle polytomique est ordonné, avec un risque d'erreur de $5 \%$. Dans le cadre de cette étude, l'approche polytomique ordonnée est bel et bien justifiée pour les modèles $2,3,4$ et 5 , eu égard aux bons résultats issus du test. La démarche, consistant à adopter ces trois spécifications (deux logits dichotomiques et un trichotomique), a pour but de mettre en exergue la robustesse des résultats obtenus, par une relative stabilité des valeurs et des signes des coefficients, quelle que soit la modélisation retenue.

\section{Des interprétations « toutes choses égales par ailleurs »}

Les dimensions explicatives sont représentées par les modalités de différentes variables. Chacune de ces modalités est introduite sous forme dichotomique. Une modalité de référence doit être choisie pour chaque variable explicative de façon à éviter le problème de colinéarité avec la constante du modèle. Le choix de la modalité (ou situation) de référence est « libre ». La seule chose à éviter est de choisir une situation de référence d'effectif trop faible car le risque est alors de détériorer la précision des estimations. Ici on a choisi des modalités de référence extrêmes, de façon à avoir le maximum de coefficients significativement non nuls, tout en respectant la remarque précédente.

Les coefficients du modèle sont estimés par la méthode de maximum de vraisemblance. Compte tenu de ce qui vient d'être dit, l'interprétation du coefficient estimé d'un facteur explicatif doit être faite «toutes choses étant égales " sur les autres facteurs et par rapport aux facteurs de référence. La $P$-value, qui correspond au test de Wald de nullité du coefficient estimé, représente la probabilité de se « tromper ». Par « tromper » on comprend le fait d'affirmer qu'un facteur explicatif aurait une influence sur la variable expliquée alors que dans la réalité l'influence serait nulle. Plus cette probabilité est faible et plus il serait légitime d'affirmer que le facteur aurait une influence sur le caractère étudié. Pour un seuil de risque fixé, on parle alors d'influence significative au seuil choisi. Dans les tableaux d'estimation, seuls les coefficients significatifs au seuil de $10 \%$ ont été reportés. Dans les tableaux résumés, les coefficients non significatifs au seuil de $5 \%$ mais significatifs au seuil de $10 \%$ sont entre parenthèses.

Le sens de l'influence d'une variable explicative sur la variable expliquée est représenté par le signe du coefficient estimé : lorsque celui-ci est positif l'influence est positive et négative dans le cas contraire.

Deux indicateurs du bon ajustement du modèle et de sa capacité prédictive sont également présentés (cf. tableaux A, B et C). II s'agit respectivement de la $P$-value correspondant au test LR («Likelyhood Ratio » pour rapport de vraisemblance) de nullité de l'ensemble des coefficients estimés (hormis la constante) et de la concordance qui représente l'association entre la valeur prédite pour la variable expliquée et sa vraie valeur dans l'échantillon (plus la concordance est proche de 1 et plus le pouvoir explicatif du modèle est important). Parallèlement, deux autres indicateurs du bon ajustement du modèle sont aussi présentés. Ils testent aussi la nullité globale des coefficients estimés ( $P$-values de Score et de Wald), afin de corroborer l'information donnée par la $P$-value LR. Plus les valeurs de ces trois $P$-values sont faibles, plus le pouvoir explicatif du modèle est élevé. Lorsque cette valeur dépasse un certain seuil $(5 \%$ ou

1. Pour plus de développements, cf. Lollivier, Marpsat et Verger (1996) dont on s'inspire dans cette présentation très résumée. 
$10 \%$ selon le degré d'exigence que l'on s'est fixé), on peut alors admettre que le modèle est mal spécifié.

Bien que les modèles estimés présentent une concordance correcte, il est certain que des facteurs explicatifs pertinents manquent. Des facteurs explicatifs sont également pris en compte de façon limitée. Par exemple, la décomposition sectorielle est pour le moins fruste, puisqu'elle ne distingue que deux secteurs (services et industrie-agriculture-construction).

Une première vérification de la robustesse des résultats des estimations a été faite en s'assurant qu'ils étaient assez stables lorsque la liste des variables explicatives est modifiée. Ainsi, dans chaque tableau de résultats détaillés des estimations, cinq ensembles de variables explicatives ont successivement été ajoutés. Une autre vérification de la robustesse a été réalisée en s'assurant que les résultats des analyses dichotomiques et de l'analyse polytomique (cf. ci-dessus) étaient cohérents. Ces deux vérifications confirment une forte robustesse des résultats obtenus. Cependant, il va de soi que les résultats présentés ici doivent être considérés avec la prudence d'usage: bien que déjà confortés par les résultats de quelques autres études, ils le seront encore davantage par une confrontation avec les analyses à venir sur ce thème.

Les tableaux présentent respectivement les résultats détaillés des analyses dichotomiques distinguant l'amélioration de la vie quotidienne à l'absence de changement ou la dégradation (cf. tableau A), ou la dégradation à l'amélioration ou l'absence de changement (cf. tableau B), et de l'analyse polytomique ordonnée distinguant ces trois modalités (cf. tableau $\mathrm{C}$ ).

Tableau A

Approche dichotomique : l'amélioration est opposée à la dégradation et à l'absence de changement (résultats détaillés des estimations logistiques)

\begin{tabular}{|c|c|c|c|c|c|c|c|c|c|c|}
\hline & \multicolumn{2}{|c|}{ Modèle 1} & \multicolumn{2}{|c|}{ Modèle 2} & \multicolumn{2}{|c|}{ Modèle 3} & \multicolumn{2}{|c|}{ Modèle 4} & \multicolumn{2}{|c|}{ Modèle 5} \\
\hline & Coeff. & Wald & Coeff. & Wald & Coeff. & Wald & Coeff. & Wald & Coeff. & Wald \\
\hline \multicolumn{11}{|c|}{ Variables d'état } \\
\hline \multicolumn{11}{|l|}{ Sexe et Enfant } \\
\hline $\begin{array}{l}\text { Femmes avec enfant de moins } \\
\text { de } 12 \text { ans }\end{array}$ & 0,63 & 7,47 & 0,57 & 5,78 & $(0,48)$ & 3,65 & & & & \\
\hline Autres femmes & Réf. & Réf. & Réf. & Réf. & Réf. & Réf. & Réf. & Réf. & Réf. & Réf. \\
\hline \multirow{2}{*}{\multicolumn{11}{|c|}{$\begin{array}{l}\text { Hommes avec enfants de moins de } \\
12 \text { ans } \\
\text { Autres hommes }\end{array}$}} \\
\hline & & & & & & & & & & \\
\hline \multicolumn{11}{|l|}{ Âge } \\
\hline 15 (borne inférieure) à 29 ans & Réf. & Réf. & Réf. & Réf. & Réf. & Réf. & Réf. & Réf. & Réf. & Réf. \\
\hline 30 à 39 ans & & & & & & & & & & \\
\hline 40 à 49 ans & & & & & & & & & & \\
\hline 50 ans et plus & & & & & & & & & & \\
\hline \multicolumn{11}{|l|}{ Diplôme } \\
\hline Aucun diplôme & Réf. & Réf. & Réf. & Réf. & Réf. & Réf. & Réf. & Réf. & Réf. & Réf. \\
\hline Enseignement court & 0,49 & 4,40 & & & & & & & & \\
\hline Niveau secondaire & $(0,49)$ & 3,41 & & & & & & & & \\
\hline Enseignement supérieur & 0,66 & 6,02 & $(0,53)$ & 3,61 & & & & & & \\
\hline \multicolumn{11}{|l|}{ Catégorie professionnelle } \\
\hline Employé ou ouvrier non qualifié & Réf. & Réf. & Réf. & Réf. & Réf. & Réf. & Réf. & Réf. & Réf. & Réf. \\
\hline Employé ou ouvrier qualifié & & & & & & & & & & \\
\hline Profession Intermédiaire & & & & & & & & & & \\
\hline Cadre & & & & & & & & & & \\
\hline \multicolumn{11}{|l|}{ Revenu mensuel du ménage } \\
\hline Moins de 10000 francs & Réf. & Réf. & Réf. & Réf. & Réf. & Réf. & Réf. & Réf. & Réf. & Réf. \\
\hline De 10001 à 15000 francs & 0,36 & 5,01 & $(0,32)$ & 3,78 & $(0,32)$ & 3,30 & & & & \\
\hline De 15001 à 20000 francs & 0,67 & 14,02 & 0,68 & 13,20 & 0,66 & 10,98 & 0,48 & 5,09 & 0,52 & 5,44 \\
\hline De 20001 à 25000 francs & 1,08 & 21,52 & 1,08 & 20,11 & 1,05 & 16,86 & 0,69 & 6,22 & 0,67 & 5,56 \\
\hline Plus de 25000 francs & 1,13 & 18,06 & 1,16 & 17,74 & 1,11 & 14,46 & 0,80 & 6,45 & 0,86 & 6,94 \\
\hline \multicolumn{11}{|l|}{ Occupation du conjoint } \\
\hline Pas de conjoint & Réf. & Réf. & Réf. & Réf. & Réf. & Réf. & Réf. & Réf. & Réf. & Réf. \\
\hline Le conjoint ne travaille pas & & & & & & & $-0,99$ & 16,52 & $-0,99$ & 15,27 \\
\hline Le conjoint travaille à temps partiel & & & & & & & $-0,74$ & 7,12 & $-0,71$ & 6,25 \\
\hline
\end{tabular}


Tableau A (suite)

Approche dichotomique : l'amélioration est opposée à la dégradation et à l'absence de changement (résultats détaillés des estimations logistiques)

\begin{tabular}{|c|c|c|c|c|c|c|c|c|c|c|}
\hline & \multicolumn{2}{|c|}{ Modèle 1} & \multicolumn{2}{|c|}{ Modèle 2} & \multicolumn{2}{|c|}{ Modèle 3} & \multicolumn{2}{|c|}{ Modèle 4} & \multicolumn{2}{|c|}{ Modèle 5} \\
\hline & Coeff. & Wald & Coeff. & Wald & Coeff. & Wald & Coeff. & Wald & Coeff. & Wald \\
\hline $\begin{array}{l}\text { Le conjoint travaille à temps com- } \\
\text { plet et connaît une RTT avec modu- } \\
\text { lation } \\
\text { Le conjoint travaille à temps com- } \\
\text { plet et connaît une RTT sans modu- } \\
\text { lation } \\
\text { Le conjoint travaille à temps com- } \\
\text { plet sans RTT }\end{array}$ & $\begin{array}{l}-0,47 \\
(-0,32)\end{array}$ & 4,02 & $\begin{array}{l}-0,52 \\
(-0,37)\end{array}$ & 4,47 & $\begin{array}{l}-0,55 \\
(-0,37) \\
-0,44\end{array}$ & $\begin{array}{l}2,82 \\
4,63\end{array}$ & $\begin{array}{l}-0,97 \\
-0,78 \\
-0,82\end{array}$ & $\begin{array}{l}11,07 \\
10,12 \\
12,79\end{array}$ & $\begin{array}{l}-0,94 \\
-0,75 \\
-0,83\end{array}$ & $\begin{array}{r}9,85 \\
8,61 \\
12,28\end{array}$ \\
\hline $\begin{array}{l}\text { Type d'habitat } \\
\text { Rural } \\
\text { Multi-polarisé } \\
\text { Périurbain } \\
\text { Pôle urbain hors Paris et région } \\
\text { parisienne } \\
\text { Paris et région parisienne }\end{array}$ & 0,35 & Réf. & $\begin{array}{l}\text { Réf. } \\
0,34 \\
0,52\end{array}$ & $\begin{array}{l}\text { Réf. } \\
\\
4,53 \\
5,32\end{array}$ & $(0,46)$ & 3,60 & $\begin{array}{l}\text { Réf. } \\
(0,30) \\
(0,53)\end{array}$ & $\begin{array}{c}\text { Réf. } \\
\\
2.64 \\
3,87\end{array}$ & Réf. & $\begin{array}{l}\text { Réf. } \\
\\
\\
3,58 \\
3,37\end{array}$ \\
\hline $\begin{array}{l}\text { Temps de transport } \\
\text { Moins de } 15 \text { minutes } \\
\text { Entre } 15 \text { minutes et une demi-heure } \\
\text { Plus d'une demi-heure }\end{array}$ & Réf. & Réf. & Réf. & Réf. & Réf. & Réf. & Réf. & Réf. & Réf. & Réf. \\
\hline $\begin{array}{l}\text { Effectif de l'entreprise } \\
\text { Moins de } 51 \text { salariés } \\
\text { De } 51 \text { à } 150 \text { salariés } \\
\text { De } 151 \text { à } 550 \text { salariés } \\
\text { De } 551 \text { à } 2000 \text { salariés } \\
\text { Plus de } 2000 \text { salariés }\end{array}$ & $\begin{array}{l}\text { Réf. } \\
-0,42 \\
(-0,32)\end{array}$ & $\begin{array}{l}\text { Réf. } \\
\text { 4,85 } \\
\text { 2,80 }\end{array}$ & Réf. & Réf. & Réf. & Réf. & Réf. & Réf. & Réf. & Réf. \\
\hline \multicolumn{11}{|c|}{ Variables caractérisant l'accord RTT } \\
\hline $\begin{array}{l}\text { Type d'accord } \\
R T T \text { sans aide incitative } \\
\text { «Robien » défensif } \\
\text { « Robien » offensif } \\
\text { «Aubry } 1 \text { » défensif } \\
\text { «Aubry } 1 \text { » offensif }\end{array}$ & & & $\begin{array}{l}\text { Réf. } \\
1,07 \\
0,95 \\
1,09 \\
1,00\end{array}$ & $\begin{array}{c}\text { Réf. } \\
24,68 \\
27,11 \\
20,37 \\
42,46\end{array}$ & $\begin{array}{l}\text { Réf. } \\
1,15 \\
0,94 \\
0,93 \\
0,88\end{array}$ & $\begin{array}{c}\text { Réf. } \\
21,08 \\
20,24 \\
11,68 \\
25,01\end{array}$ & $\begin{array}{l}\text { Réf. } \\
0,75 \\
0,63 \\
0,77 \\
0,70\end{array}$ & $\begin{array}{r}\text { Réf. } \\
7,14 \\
7,37 \\
6,87 \\
12,73\end{array}$ & $\begin{array}{l}\text { Réf. } \\
0,89 \\
0,64 \\
0,73 \\
0,76\end{array}$ & \begin{tabular}{r}
\multicolumn{1}{c}{ Réf. } \\
9,12 \\
7,17 \\
5,95 \\
14,12
\end{tabular} \\
\hline $\begin{array}{l}\text { Consultation... } \\
\text {... par la direction ou l'encadre- } \\
\text { ment intermédiaire } \\
\text { Non } \\
\text { Oui } \\
\text {... par les syndicats ou les élus } \\
\text { du personnel }\end{array}$ & & & $\begin{array}{l}\text { Réf. } \\
0,23\end{array}$ & $\begin{array}{l}\text { Réf. } \\
4,06\end{array}$ & Réf. & Réf. & Réf. & Réf. & Réf. & Réf. \\
\hline $\begin{array}{l}\text { Non } \\
\text { Oui } \\
\text { W. par un ou des groupe(s) } \\
\text { de travail } \\
\text { Non } \\
\text { Oui }\end{array}$ & & & $\begin{array}{l}\text { Réf. } \\
0,39\end{array}$ & $\begin{array}{l}\text { Réf. } \\
6,45\end{array}$ & $\begin{array}{l}\text { Réf. } \\
0,37\end{array}$ & $\begin{array}{l}\text { Réf. } \\
5,10\end{array}$ & Réf. & Réf. & Réf. & $\begin{array}{l}\text { Réf. } \\
\text { Réf. }\end{array}$ \\
\hline \multicolumn{11}{|c|}{ Modifications induites par la RTT } \\
\hline $\begin{array}{l}\text { Modalités de RTT } \\
\text { - Journées de travail plus } \\
\text { courtes }\end{array}$ & & & & & & & & & & \\
\hline $\begin{array}{l}\text { Non } \\
\text { Oui } \\
\text { - Demi-journée ou jour non tra- } \\
\text { vaillé chaque semaine, tous les } \\
15 \text { jours ou tous les mois }\end{array}$ & & & & & $\begin{array}{l}\text { Réf. } \\
(0,25)\end{array}$ & $\begin{array}{l}\text { Réf. } \\
2,75\end{array}$ & $\begin{array}{l}\text { Réf. } \\
0,43\end{array}$ & $\begin{array}{l}\text { Réf. } \\
6,52\end{array}$ & $\begin{array}{l}\text { Réf. } \\
0,44\end{array}$ & $\begin{array}{l}\text { Réf. } \\
6,33\end{array}$ \\
\hline $\begin{array}{l}\text { Non } \\
\text { Oui }\end{array}$ & & & & & $\begin{array}{l}\text { Réf. } \\
0,90\end{array}$ & $\begin{array}{c}\text { Réf. } \\
31,14\end{array}$ & $\begin{array}{l}\text { Réf. } \\
0,74\end{array}$ & $\begin{array}{c}\text { Réf. } \\
17,82\end{array}$ & $\begin{array}{l}\text { Réf. } \\
0,70\end{array}$ & $\begin{array}{c}\text { Réf. } \\
15,07\end{array}$ \\
\hline
\end{tabular}


Tableau A (suite)

Approche dichotomique : l'amélioration est opposée à la dégradation et à l'absence de changement (résultats détaillés des estimations logistiques)

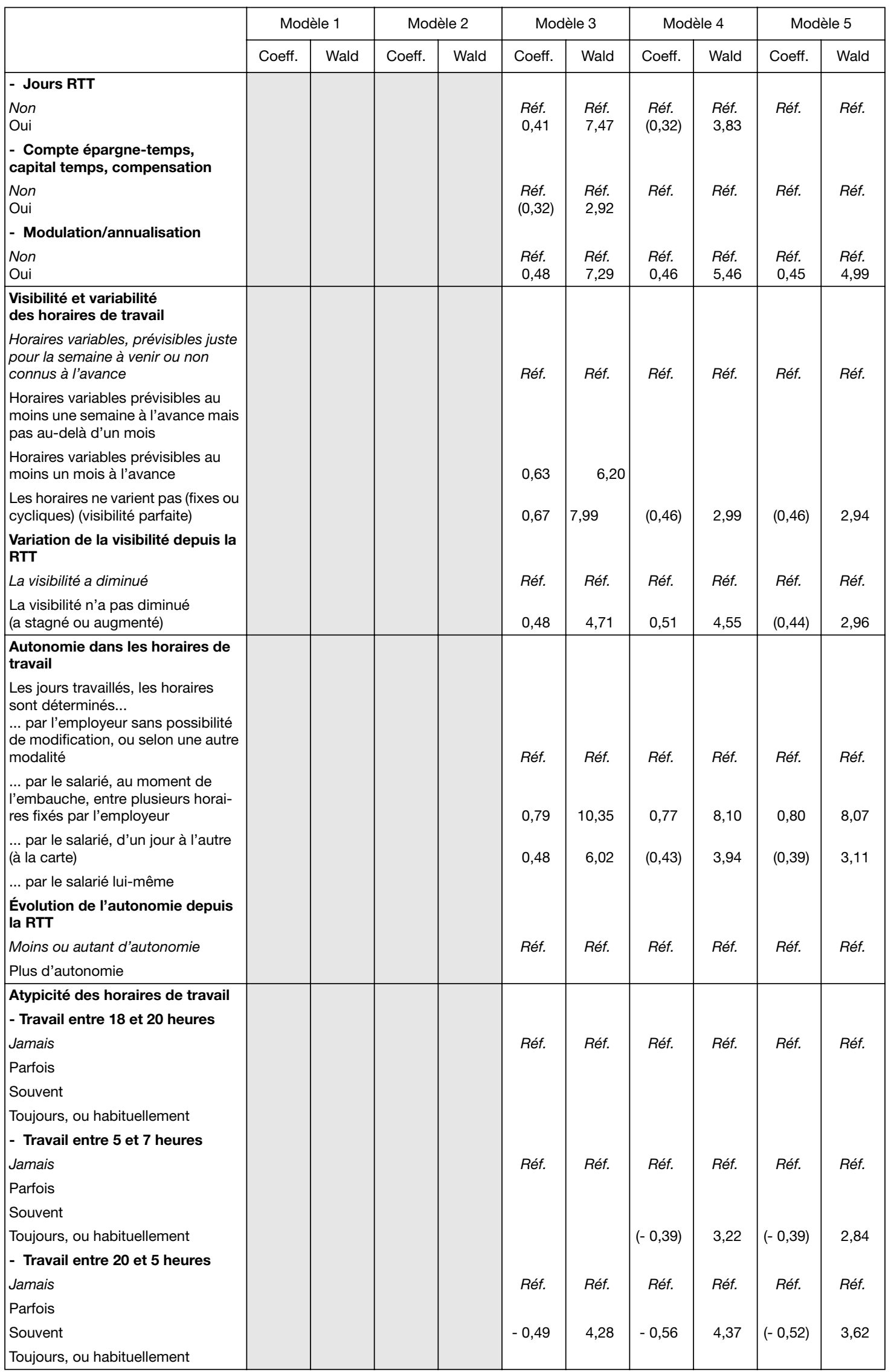


Tableau A (suite)

Approche dichotomique : l'amélioration est opposée à la dégradation et à l'absence de changement (résultats détaillés des estimations logistiques)

\begin{tabular}{|c|c|c|c|c|c|c|c|c|c|c|}
\hline & \multicolumn{2}{|c|}{ Modèle 1} & \multicolumn{2}{|c|}{ Modèle 2} & \multicolumn{2}{|c|}{ Modèle 3} & \multicolumn{2}{|c|}{ Modèle 4} & \multicolumn{2}{|c|}{ Modèle 5} \\
\hline & Coeff. & Wald & Coeff. & Wald & Coeff. & Wald & Coeff. & Wald & Coeff. & Wald \\
\hline $\begin{array}{l}\text { Évolution de l'atypicité depuis la } \\
\text { RTT } \\
\text { Moins d'atypicité } \\
\text { Pas moins d'atypicité ( autant ou } \\
\text { plus ) }\end{array}$ & & & & & $\begin{array}{c}\text { Réf. } \\
(-0,62)\end{array}$ & $\begin{array}{l}\text { Réf. } \\
3,48\end{array}$ & $\begin{array}{l}\text { Réf. } \\
(0,59)\end{array}$ & $\begin{array}{l}\text { Réf. } \\
2,57\end{array}$ & Réf. & Réf. \\
\hline $\begin{array}{l}\text { La durée effective du travail cor- } \\
\text { respond-elle à ce qui est prévu } \\
\text { dans l'accord? } \\
\text { Non } \\
\text { Oui }\end{array}$ & & & & & $\begin{array}{l}\text { Réf. } \\
0,79\end{array}$ & $\begin{array}{c}\text { Réf. } \\
31,23\end{array}$ & $\begin{array}{l}\text { Réf. } \\
0,63\end{array}$ & $\begin{array}{c}\text { Réf. } \\
16,4\end{array}$ & $\begin{array}{l}\text { Réf. } \\
0,63\end{array}$ & $\begin{array}{c}\text { Réf. } \\
15,35\end{array}$ \\
\hline $\begin{array}{l}\text { Conditions de travail } \\
\text { - Délais fixés } \\
\text { Non-raccourcissement des délais } \\
\text { Les délais ont été raccourcis } \\
\text { - Objectifs fixés } \\
\text { Non-augmentation des objectifs } \\
\text { Les objectifs ont été augmentés }\end{array}$ & & & & & $\begin{array}{l}\text { Réf. } \\
\text { Réf. }\end{array}$ & $\begin{array}{l}\text { Réf. } \\
\text { Réf. }\end{array}$ & $\begin{array}{l}\text { Réf. } \\
\text { Réf. }\end{array}$ & $\begin{array}{l}\text { Réf. } \\
\text { Réf. }\end{array}$ & $\begin{array}{l}\text { Réf. } \\
\text { Réf. }\end{array}$ & $\begin{array}{l}\text { Réf. } \\
\text { Réf. }\end{array}$ \\
\hline $\begin{array}{l}\text { Effet de la RTT } \\
\text { sur la rémunération } \\
\text { Aucune conséquence } \\
\text { Baisse de la rémunération } \\
\text { Gel de la rémunération } \\
\text { Modération plus forte ou hausse } \\
\text { moins forte que prévu }\end{array}$ & & & & & $\begin{array}{c}\text { Réf. } \\
(-0,34)\end{array}$ & $\begin{array}{l}\text { Réf. } \\
2,80\end{array}$ & $\begin{array}{c}\text { Réf. } \\
-0,50\end{array}$ & $\begin{array}{l}\text { Réf. } \\
4,87\end{array}$ & $\begin{array}{c}\text { Réf. } \\
-0,56 \\
(-0,26)\end{array}$ & $\begin{array}{l}\text { Réf. } \\
5,67 \\
2,74\end{array}$ \\
\hline & & Usages & Iu temp & dégagé & par la RT & & & & & \\
\hline $\begin{array}{l}\text { Passer plus de temps avec sa } \\
\text { famille } \\
\text { Non } \\
\text { Oui }\end{array}$ & & & & & & & $\begin{array}{l}\text { Réf. } \\
1,34\end{array}$ & $\begin{array}{c}\text { Réf. } \\
87,14\end{array}$ & $\begin{array}{l}\text { Réf. } \\
1,21\end{array}$ & $\begin{array}{c}\text { Réf. } \\
67,27\end{array}$ \\
\hline $\begin{array}{l}\text { Activités domestiques, dont : } \\
\text { - Faire plus de jardinage } \\
\text { ou de bricolage } \\
\text { Non } \\
\text { Oui } \\
\text { - Passer plus de temps à au } \\
\text { moins une autre activité } \\
\text { domestique } \\
\text { Non } \\
\text { Oui }\end{array}$ & & & & & & & $\begin{array}{l}\text { Réf. } \\
0,64\end{array}$ & $\begin{array}{l}\text { Réf. } \\
4,65 \\
\\
\text { Réf. } \\
8,05\end{array}$ & $\begin{array}{l}\text { Réf. } \\
(0,34) \\
\text { Réf. } \\
0,52\end{array}$ & $\begin{array}{l}\text { Réf. } \\
2,65 \\
\text { Réf. } \\
5,06\end{array}$ \\
\hline $\begin{array}{l}\text { Loisirs } \\
\text { - Passer plus de temps à se } \\
\text { reposer } \\
\text { Non } \\
\text { Oui } \\
\text { - Passer davantage de temps à } \\
\text { au moins une activité de loisir } \\
\text { autre que le repos } \\
\text { Non } \\
\text { Oui }\end{array}$ & & & & & & & $\begin{array}{l}\text { Réf. } \\
0,52 \\
\text { Réf. } \\
0,73\end{array}$ & $\begin{array}{c}\text { Réf. } \\
12,70 \\
\\
\text { Réf. } \\
28,63\end{array}$ & $\begin{array}{l}\text { Réf. } \\
0,49 \\
\text { Réf. } \\
0,66\end{array}$ & $\begin{array}{c}\text { Réf. } \\
10,41 \\
\\
\text { Réf. } \\
22,54\end{array}$ \\
\hline Que & IOns « & jet & " con & nant & Siode & écéda & a RTT & & & \\
\hline $\begin{array}{l}\text { Réflexion sur l'utilisation du } \\
\text { temps } \\
\text { N'avait pas réfléchi } \\
\text { Avait réfléchi mais n'a pas utilisé le } \\
\text { temps comme souhaité } \\
\text { Avait réfléchi et a utilisé le temps } \\
\text { comme souhaité }\end{array}$ & & & & & & & & & $\begin{array}{c}\text { Réf. } \\
-0,63 \\
0,71\end{array}$ & $\begin{array}{r}\text { Réf. } \\
8,23 \\
17,98\end{array}$ \\
\hline
\end{tabular}


Tableau A (fin)

Approche dichotomique : l'amélioration est opposée à la dégradation et à l'absence de changement (résultats détaillés des estimations logistiques)

\begin{tabular}{|c|c|c|c|c|c|c|c|c|c|c|}
\hline & \multicolumn{2}{|c|}{ Modèle 1} & \multicolumn{2}{|c|}{ Modèle 2} & \multicolumn{2}{|c|}{ Modèle 3} & \multicolumn{2}{|c|}{ Modèle 4} & \multicolumn{2}{|c|}{ Modèle 5} \\
\hline & Coeff. & Wald & Coeff. & Wald & Coeff. & Wald & Coeff. & Wald & Coeff. & Wald \\
\hline \multicolumn{11}{|c|}{ Impression de manquer de temps } \\
\hline Jamais & & & & & & & & & Réf. & Réf. \\
\hline Rarement & & & & & & & & & 0,61 & 6,46 \\
\hline Parfois & & & & & & & & & 0,58 & 10,67 \\
\hline Souvent & & & & & & & & & 1,16 & 32,58 \\
\hline Toujours & & & & & & & & & 0,73 & 12,13 \\
\hline Constante & & & $-1,69$ & 16,3 & $-3,47$ & 36,38 & $-3,65$ & 33,12 & $-4,22$ & 40,02 \\
\hline Nombre d'observations & \multicolumn{2}{|c|}{1618} & \multicolumn{2}{|c|}{1618} & \multicolumn{2}{|c|}{1618} & \multicolumn{2}{|c|}{1618} & \multicolumn{2}{|c|}{1618} \\
\hline P-value LR & \multicolumn{2}{|c|}{$<0,0001$} & \multicolumn{2}{|c|}{$<0,0001$} & \multicolumn{2}{|c|}{$<0,0001$} & \multicolumn{2}{|c|}{$<0,0001$} & \multicolumn{2}{|c|}{$<0,0001$} \\
\hline P-value Score & \multicolumn{2}{|c|}{$<0,0001$} & \multicolumn{2}{|c|}{$<0,0001$} & \multicolumn{2}{|c|}{$<0,0001$} & \multicolumn{2}{|c|}{$<0,0001$} & \multicolumn{2}{|c|}{$<0,0001$} \\
\hline P-value Wald & \multicolumn{2}{|c|}{$<0,0001$} & \multicolumn{2}{|c|}{$<0,0001$} & \multicolumn{2}{|c|}{$<0,0001$} & \multicolumn{2}{|c|}{$<0,0001$} & \multicolumn{2}{|c|}{$<0,0001$} \\
\hline Concordance & \multicolumn{2}{|c|}{63,1} & \multicolumn{2}{|c|}{67,9} & \multicolumn{2}{|c|}{75,0} & \multicolumn{2}{|c|}{82,8} & \multicolumn{2}{|c|}{84,4} \\
\hline
\end{tabular}

Les situations de référence dans les estimations sont indiquées par « Réf. ».

Les 5 modèles se distinguent par un nombre croissant de variables explicatives retenues. Ainsi, les variables explicatives retenues sont : dans le modèle 1, les seules variables d'état; dans le modèle 2, les variables explicatives du modèle 1 et les variables caractérisant l'accord; dans le modèle 3, les variables explicatives du modèle 2 et les variables indiquant les modifications induites par la RTT; dans le modèle 4, les variables explicatives du modèle 3 et les variables indiquant les usages du temps dégagé par la RTT; dans le modèle 5 , les variables explicatives du modèle 4 et les variables de réponses aux questions "subjectives " concernant la période précédant l'accord.

La statistique de Wald est le carré de la statistique de Student. Si elle dépasse 4, cela signifie que le coefficient est significatif au seuil de $5 \%$. Les coefficients non significatifs ne sont pas indiqués afin d'alléger le tableau. Mais les coefficients proches (à un seuil compris entre $5 \%$ et $10 \%)$ sont cependant fournis entre parenthèses.

Lecture : dans le cadre du modèle 2, pour la rubrique "Type d'accord », les autres modalités se distinguent significativement au seuil de $5 \%$ des $R T T$ sans aide incitative retenus comme référence. Les salariés présentant un accord de RTT avec aide incitative ont ainsi une probabilité d'avoir connu, via la RTT, une amélioration de leur vie quotidienne significativement supérieure à ceux dont l'accord de $R T T$ ne s'accompagne pas d'une telle aide.

Source des données de base : enquête RTT et Modes de vie, Dares.

Tableau B

Approche dichotomique : la dégradation est opposée à l'amélioration et à l'absence de changement (résultats détaillés des estimations logistiques)

\begin{tabular}{|c|c|c|c|c|c|c|c|c|c|c|}
\hline & \multicolumn{2}{|c|}{ Modèle 1} & \multicolumn{2}{|c|}{ Modèle 2} & \multicolumn{2}{|c|}{ Modèle 3} & \multicolumn{2}{|c|}{ Modèle 4} & \multicolumn{2}{|c|}{ Modèle 5} \\
\hline & Coeff. & Wald & Coeff. & Wald & Coeff. & Wald & Coeff. & Wald & Coeff. & Wald \\
\hline \multicolumn{11}{|c|}{ Variables d'état } \\
\hline Sexe et Enfant & & & & & & & & & & \\
\hline $\begin{array}{l}\text { Femmes avec enfant de moins de } \\
12 \text { ans }\end{array}$ & $-1,31$ & 9,06 & $-1,27$ & 8,43 & $-1,29$ & 7,30 & $-1,09$ & 5,13 & $-1,21$ & 5,73 \\
\hline Autres femmes & Réf. & Réf. & Réf. & Réf. & Réf. & Réf. & Réf. & Réf. & Réf. & Réf. \\
\hline $\begin{array}{l}\text { Hommes avec enfants de moins de } \\
12 \text { ans }\end{array}$ & & & & & & & & & & \\
\hline \multicolumn{11}{|l|}{ Autres hommes } \\
\hline Âge & & & & & & & & & & \\
\hline 15 (borne inférieure) à 29 ans & Réf. & Réf. & Réf. & Réf. & Réf. & Réf. & Réf. & Réf. & Réf. & Réf. \\
\hline 30 à 39 ans & & & & & & & & & & \\
\hline 40 à 49 ans & & & & & & & & & & \\
\hline 50 ans et plus & & & & & & & & & & \\
\hline \multicolumn{11}{|l|}{ Diplôme } \\
\hline Aucun diplôme & Réf. & Réf. & Réf. & Réf. & Réf. & Réf. & Réf. & Réf. & Réf. & Réf. \\
\hline Enseignement court & $(-0,68)$ & 3,25 & $(-0,72)$ & 3,60 & $-0,92$ & 5,10 & $-0,94$ & 5,24 & $(-0,82)$ & 3,96 \\
\hline Niveau secondaire & $-0,86$ & 4,08 & $-0,91$ & 4,48 & $-1,25$ & 7,34 & $-1,28$ & 7,54 & $-1,13$ & 5,76 \\
\hline Enseignement supérieur & & & & & & & & & & \\
\hline
\end{tabular}


Tableau B (suite)

Approche dichotomique : la dégradation est opposée à l'amélioration et à l'absence de changement (résultats détaillés des estimations logistiques)

\begin{tabular}{|c|c|c|c|c|c|c|c|c|c|c|}
\hline & \multicolumn{2}{|c|}{ Modèle 1} & \multicolumn{2}{|c|}{ Modèle 2} & \multicolumn{2}{|c|}{ Modèle 3} & \multicolumn{2}{|c|}{ Modèle 4} & \multicolumn{2}{|c|}{ Modèle 5} \\
\hline & Coeff. & Wald & Coeff. & Wald & Coeff. & Wald & Coeff. & Wald & Coeff. & Wald \\
\hline $\begin{array}{l}\text { Catégorie professionnelle } \\
\text { Employé ou ouvrier non qualifié } \\
\text { Employé ou ouvrier qualifié } \\
\text { Profession Intermédiaire } \\
\text { Cadre }\end{array}$ & Réf. & Réf. & Réf. & Réf. & $\begin{array}{l}\text { Réf. } \\
(-0,93)\end{array}$ & $\begin{array}{l}\text { Réf. } \\
3,96\end{array}$ & $\begin{array}{l}\text { Réf. } \\
(-0,94)\end{array}$ & $\begin{array}{l}\text { Réf. } \\
3,89\end{array}$ & $\begin{array}{r}\text { Réf. } \\
-1,08\end{array}$ & $\begin{array}{l}\text { Réf. } \\
4,95\end{array}$ \\
\hline $\begin{array}{l}\text { Revenu mensuel du ménage } \\
\text { Moins de } 10000 \text { francs } \\
\text { De } 10001 \text { à } 15000 \text { francs } \\
\text { De } 15001 \text { à } 20000 \text { francs } \\
\text { De } 20001 \text { à } 25000 \text { francs } \\
\text { Plus de } 25000 \text { francs }\end{array}$ & $\begin{array}{l}\text { Réf. } \\
-0,60 \\
(-0,72)\end{array}$ & $\begin{array}{l}\text { Réf. } \\
5,55 \\
3,15\end{array}$ & $\begin{array}{l}\text { Réf. } \\
-0,57 \\
(-0,72)\end{array}$ & $\begin{array}{l}\text { Réf. } \\
4,98 \\
3,05\end{array}$ & $\begin{array}{l}\text { Réf. } \\
(-0,53)\end{array}$ & $\begin{array}{l}\text { Réf. } \\
3,67\end{array}$ & Réf. & Réf. & Réf. & Réf. \\
\hline $\begin{array}{l}\text { Occupation du conjoint } \\
\text { Pas de conjoint } \\
\text { Le conjoint ne travaille pas } \\
\text { Le conjoint travaille à temps partiel } \\
\text { Le conjoint travaille à temps com- } \\
\text { plet et connaît une RTT avec modu- } \\
\text { lation } \\
\text { Le conjoint travaille à temps com- } \\
\text { plet et connaît une RTT sans modu- } \\
\text { lation } \\
\text { Le conjoint travaille à temps com- } \\
\text { plet sans RTT }\end{array}$ & Réf. & Réf. & Réf. & Réf. & Réf. & Réf. & $\begin{array}{l}\text { Réf. } \\
(0,62)\end{array}$ & $\begin{array}{l}\text { Réf. } \\
3,82\end{array}$ & $\begin{array}{r}\text { Réf. } \\
(0,64) \\
\\
0,85\end{array}$ & $\begin{array}{l}\text { Réf. } \\
3,87\end{array}$ \\
\hline $\begin{array}{l}\text { Type d'habitat } \\
\text { Rural } \\
\text { Multi-polarisé } \\
\text { Périurbain } \\
\text { Pôle urbain hors Paris et région } \\
\text { parisienne } \\
\text { Paris et région parisienne }\end{array}$ & Réf. & Réf. & Réf. & Réf. & Réf. & Réf. & Réf. & Réf. & Réf. & Réf. \\
\hline $\begin{array}{l}\text { Temps de transport } \\
\text { Moins de } 15 \text { minutes } \\
\text { Entre } 15 \text { minutes et une demi-heure } \\
\text { Plus d'une demi-heure }\end{array}$ & $\begin{array}{l}\text { Réf. } \\
(0,38)\end{array}$ & $\begin{array}{l}\text { Réf. } \\
2,84\end{array}$ & Réf. & Réf. & Réf. & Réf. & Réf. & Réf. & Réf. & Réf. \\
\hline $\begin{array}{l}\text { Effectif de l'entreprise } \\
\text { Moins de } 51 \text { salariés } \\
\text { De } 51 \text { à } 150 \text { salariés } \\
\text { De } 151 \text { à } 550 \text { salariés } \\
\text { De } 551 \text { à } 2000 \text { salariés } \\
\text { Plus de } 2000 \text { salariés }\end{array}$ & 0,61 & 4,25 & $\begin{array}{r}\text { Réf. } \\
(0,54)\end{array}$ & $\begin{array}{l}\text { Réf. } \\
3,08\end{array}$ & Réf. & Réf. & Réf. & Réf. & Réf. & Réf. \\
\hline & & Variabl & s caract & isant l'c & ccord RT & & & & & \\
\hline $\begin{array}{l}\text { Type d'accord } \\
R T T \text { sans aide incitative } \\
\text { «Robien » défensif } \\
\text { «Robien » offensif } \\
\text { «Aubry } 1 \text { » défensif } \\
\text { «Aubry } 1 \text { » offensif } \\
\end{array}$ & & & Réf. & Réf. & $\begin{array}{c}\text { Réf. } \\
(-0,63)\end{array}$ & $\begin{array}{l}\text { Réf. } \\
3,25\end{array}$ & Réf. & Réf. & Réf. & Réf. \\
\hline $\begin{array}{l}\text { Consultation... } \\
\ldots \text {.. par la direction ou l'encadre- } \\
\text { ment intermédiaire }\end{array}$ & & & & & & & & & & \\
\hline $\begin{array}{l}\text { Non } \\
\text { Oui } \\
\text {... par les syndicats ou les élus du } \\
\text { personnel }\end{array}$ & & & Réf. & Réf. & Réf. & Réf. & Réf. & Réf. & Réf. & Réf. \\
\hline $\begin{array}{l}\text { Non } \\
\text { Oui } \\
\text {... par un ou des groupe(s) } \\
\text { de travail }\end{array}$ & & & Réf. & Réf. & Réf. & Réf. & Réf. & Réf. & Réf. & Réf. \\
\hline $\begin{array}{l}\text { Non } \\
\text { Oui }\end{array}$ & & & Réf. & Réf. & Réf. & Réf. & Réf. & Réf. & Réf. & Réf. \\
\hline
\end{tabular}


Tableau B (suite)

Approche dichotomique : la dégradation est opposée à l'amélioration et à l'absence de changement (résultats détaillés des estimations logistiques)

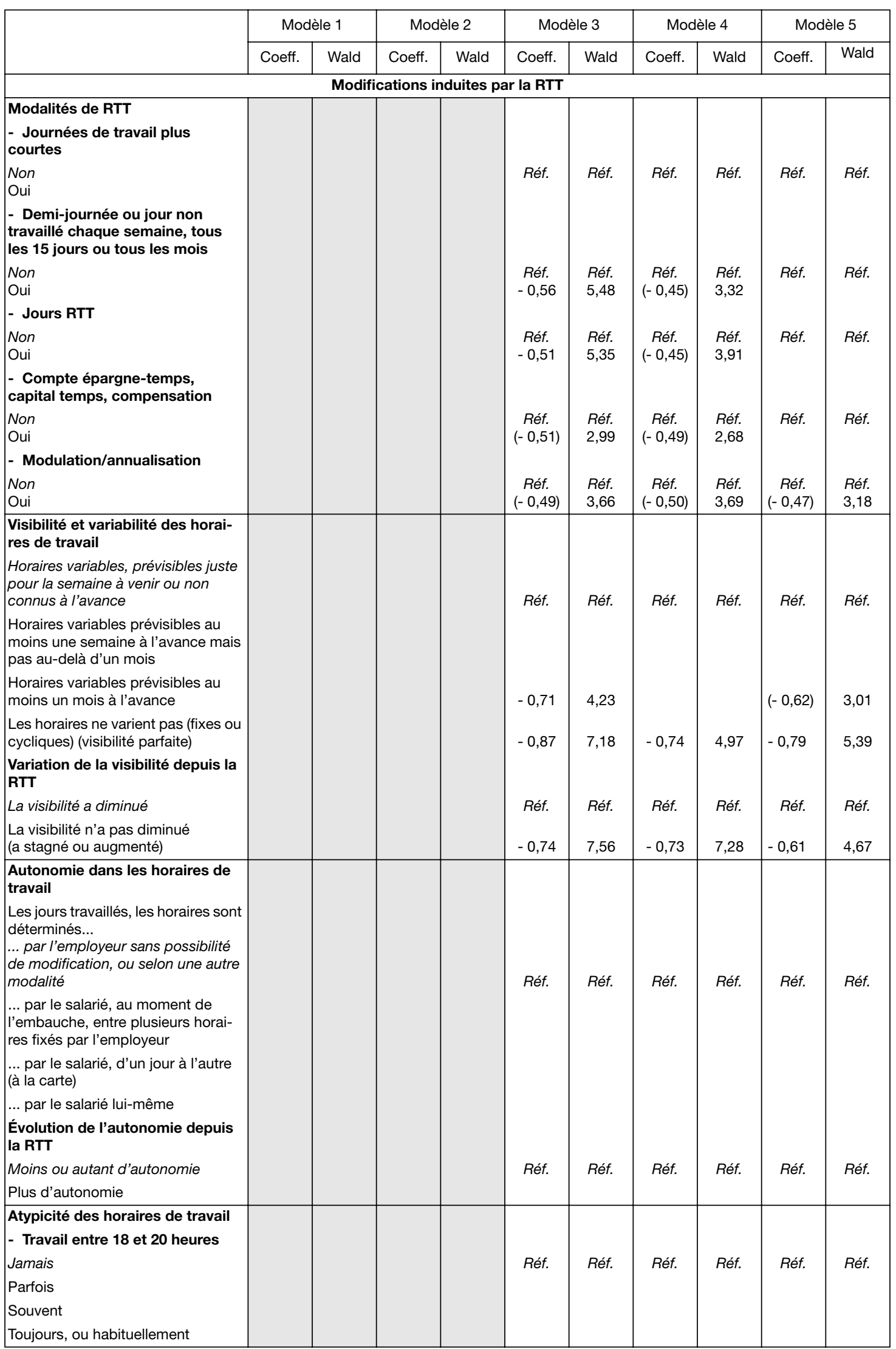


Tableau B (suite)

Approche dichotomique : la dégradation est opposée à l'amélioration et à l'absence de changement (résultats détaillés des estimations logistiques)

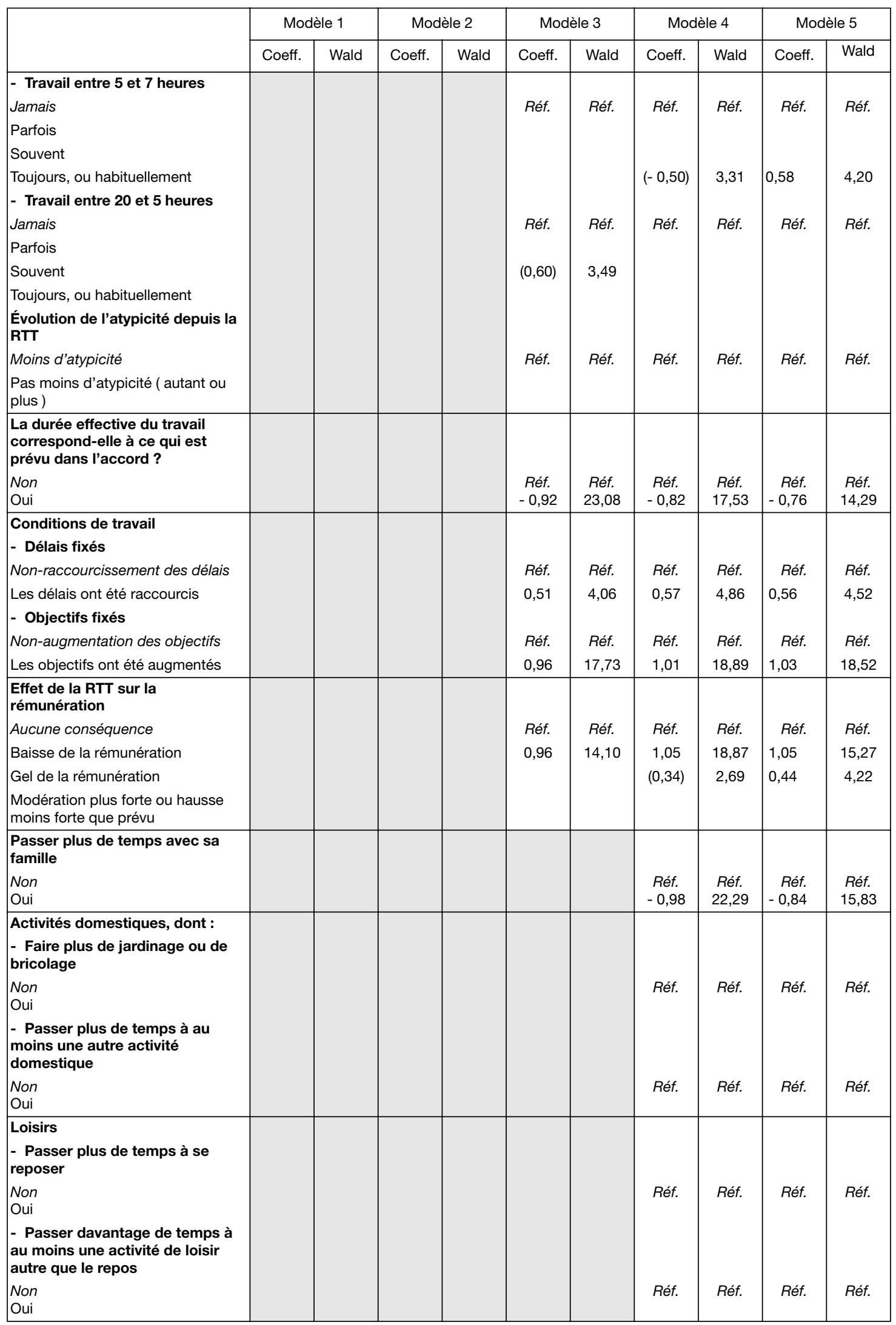


Tableau B (fin)

Approche dichotomique : la dégradation est opposée à l'amélioration et à l'absence de changement (résultats détaillés des estimations logistiques)

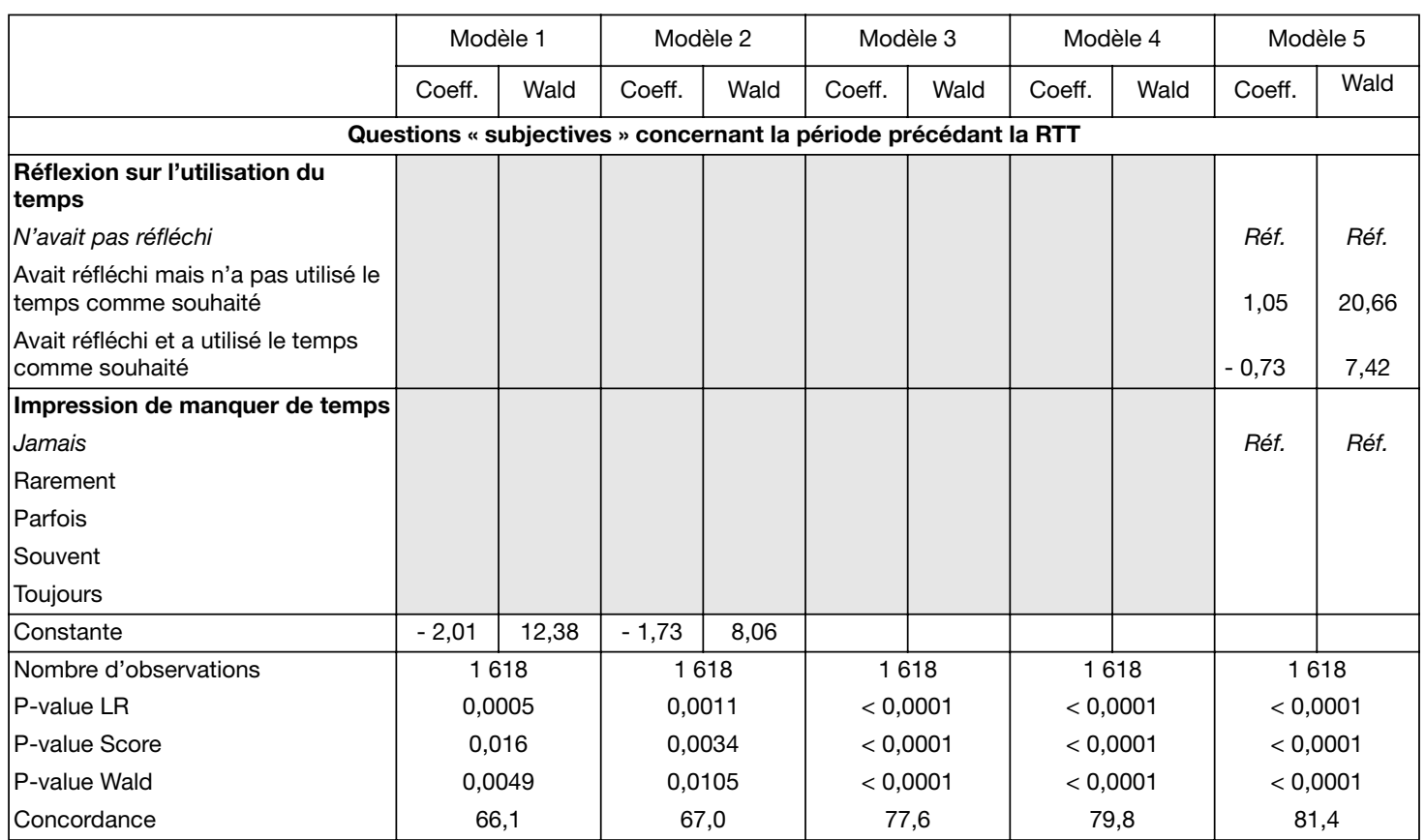

Les situations de référence dans les estimations sont indiquées par « Réf. ».

Les 5 modèles se distinguent par un nombre croissant de variables explicatives retenues. Ainsi, les variables explicatives retenues sont : dans le modèle 1, les seules variables d'état; dans le modèle 2, les variables explicatives du modèle 1 et les variables caractérisant l'accord; dans le modèle 3, les variables explicatives du modèle 2 et les variables indiquant les modifications induites par la RTT; dans le modèle 4, les variables explicatives du modèle 3 et les variables indiquant les usages du temps dégagé par la RTT; dans le modèle 5 , les variables explicatives du modèle 4 et les variables de réponses aux questions "subjectives " concernant la période précédant l'accord.

La statistique de Wald est le carré de la statistique de Student. Si elle dépasse 4, cela signifie que le coefficient est significatif au seuil de $5 \%$. Les coefficients non significatifs ne sont pas indiqués afin d'alléger le tableau. Mais les coefficients proches (à un seuil compris entre $5 \%$ et $10 \%$ ) sont cependant fournis entre parenthèses.

Lecture: pour les modèles 3, 4 et 5, la modalité relative à une augmentation des objectifs depuis la RTT se démarque significativement au seuil de $5 \%$ de la modalité correspondant au cas où les objectifs n'ont pas augmenté. Autrement dit, les salariés dont les objectifs ont été augmentés ont donc une probabilité d'afficher un sentiment de dégradation de leur vie quotidienne significativement supérieur (au seuil de $5 \%$ ) à celle qui prévaut pour les salariés n'ayant pas connu d'augmentation de leurs objectifs (pris en référence). Source des données de base : enquête RTT et Modes de vie, Dares. 
Tableau C

Approche polytomique distinguant l'amélioration, l'absence de changement et la dégradation (résultats détaillés des estimations logistiques)

\begin{tabular}{|c|c|c|c|c|c|c|c|c|c|c|}
\hline & \multicolumn{2}{|c|}{ Modèle 1} & \multicolumn{2}{|c|}{ Modèle 2} & \multicolumn{2}{|c|}{ Modèle 3} & \multicolumn{2}{|c|}{ Modèle 4} & \multicolumn{2}{|c|}{ Modèle 5} \\
\hline & Coeff. & Wald & Coeff. & Wald & Coeff. & Wald & Coeff. & Wald & Coeff. & Wald \\
\hline \multicolumn{11}{|c|}{ Variables d'état } \\
\hline \multicolumn{11}{|l|}{ Sexe et enfant } \\
\hline $\begin{array}{l}\text { Femmes avec enfant de moins de } \\
12 \text { ans }\end{array}$ & 0,69 & 9,42 & 0,63 & 7,62 & 0,57 & 5,67 & & & & \\
\hline Autres femmes & Réf. & Réf. & Réf. & Réf. & Réf. & Réf. & Réf. & Réf. & Réf. & Réf. \\
\hline $\begin{array}{l}\text { Hommes avec enfants de moins } \\
\text { de } 12 \text { ans }\end{array}$ & & & & & & & & & & \\
\hline \multicolumn{11}{|l|}{ Autres hommes } \\
\hline Âge & & & & & & & & & & \\
\hline 15 (borne inférieure) à 29 ans & Réf. & Réf. & Réf. & Réf. & Réf. & Réf. & Réf. & Réf. & Réf. & Réf. \\
\hline 30 à 39 ans & & & & & & & & & & \\
\hline 40 à 49 ans & & & & & & & & & & \\
\hline 50 ans et plus & & & & & & & & & & \\
\hline \multicolumn{11}{|l|}{ Diplôme } \\
\hline Aucun diplôme & Réf. & Réf. & Réf. & Réf. & Réf. & Réf. & Réf. & Réf. & Réf. & Réf. \\
\hline Enseignement court & & & & & & & & & & \\
\hline Niveau secondaire & & & & & & & & & & \\
\hline Enseignement supérieur & $(0,43)$ & 2,82 & & & & & & & & \\
\hline Catégorie professionnelle & & & & & & & & & & \\
\hline Employé ou ouvrier non qualifié & Réf. & Réf. & Réf. & Réf. & Réf. & Réf. & Réf. & Réf. & Réf. & Réf. \\
\hline Employé ou ouvrier qualifié & & & & & & & & & & \\
\hline Profession Intermédiaire & & & & & & & & & & \\
\hline Cadre & & & & & & & & & & \\
\hline \multicolumn{11}{|l|}{ Revenu mensuel du ménage } \\
\hline Moins de 10000 francs & Réf. & Réf. & Réf. & Réf. & Réf. & Réf. & Réf. & Réf. & Réf. & Réf. \\
\hline De 10001 à 15000 francs & 0,35 & 5,18 & $(0,30)$ & 3,82 & $(0,31)$ & 3,62 & & & & \\
\hline De 15001 à 20000 francs & 0,64 & 14,09 & 0,62 & 12,92 & 0,60 & 10,73 & 0,44 & 5,43 & 0,46 & 5,88 \\
\hline De 20001 à 25000 francs & 0,98 & 19,07 & 0,95 & 17,49 & 0,89 & 14,06 & 0,56 & 5,17 & 0,53 & 4,43 \\
\hline Plus de 25000 francs & 1,04 & 16,44 & 1,03 & 15,44 & 0,95 & 11,99 & 0,63 & 4,97 & 0,66 & 5,11 \\
\hline \multicolumn{11}{|l|}{ Occupation du conjoint } \\
\hline Pas de conjoint & Réf. & Réf. & Réf. & Réf. & Réf. & Réf. & Réf. & Réf. & Réf. & Réf. \\
\hline Le conjoint ne travaille pas & & & & & & & $-0,76$ & 12,63 & $-0,75$ & 11,89 \\
\hline Le conjoint travaille à temps partiel & & & & & & & $-0,53$ & 4,81 & $-0,50$ & 4,01 \\
\hline Le conjoint travaille à temps & & & & & & & & & & \\
\hline avec modulation & $-0,48$ & 4,50 & $-0,54$ & 5,53 & $-0,53$ & 4,95 & $-0,84$ & 11,05 & $-0,80$ & 9,62 \\
\hline Le conjoint travaille à temps & & & & & & & & & & \\
\hline sans modulation & & & & & & & $-0,56$ & 6,67 & $-0,48$ & 4,74 \\
\hline $\begin{array}{l}\text { Le conjoint travaille à temps } \\
\text { complet sans RTT }\end{array}$ & & & $(-0,31)$ & 2,95 & $(-0,37)$ & 3,76 & $-0,63$ & 9,86 & $-0,64$ & 9,97 \\
\hline \multicolumn{11}{|l|}{ Type d'habitat } \\
\hline Rural & Réf. & Réf. & Réf. & Réf. & Réf. & Réf. & Réf. & Réf. & Réf. & Réf. \\
\hline Multi-polarisé & & & & & & & & & & \\
\hline Périurbain & & & & & & & & & & \\
\hline $\begin{array}{l}\text { Pôle urbain hors Paris et région } \\
\text { parisienne }\end{array}$ & 0,30 & 4,01 & $(0,27)$ & 3,27 & & & & & & \\
\hline Paris et région parisienne & & & $(0,39)$ & 3,31 & & & & & & \\
\hline \multicolumn{11}{|l|}{ Temps de transport } \\
\hline Moins de 15 minutes & Réf. & Réf. & Réf. & Réf. & Réf. & Réf. & Réf. & Réf. & Réf. & Réf. \\
\hline Entre 15 minutes et une demi-heure & & & & & & & & & & \\
\hline Plus d'une demi-heure & & & & & & & & & & \\
\hline
\end{tabular}


Tableau C (suite)

Approche polytomique distinguant l'amélioration, l'absence de changement et la dégradation (résultats détaillés des estimations logistiques)

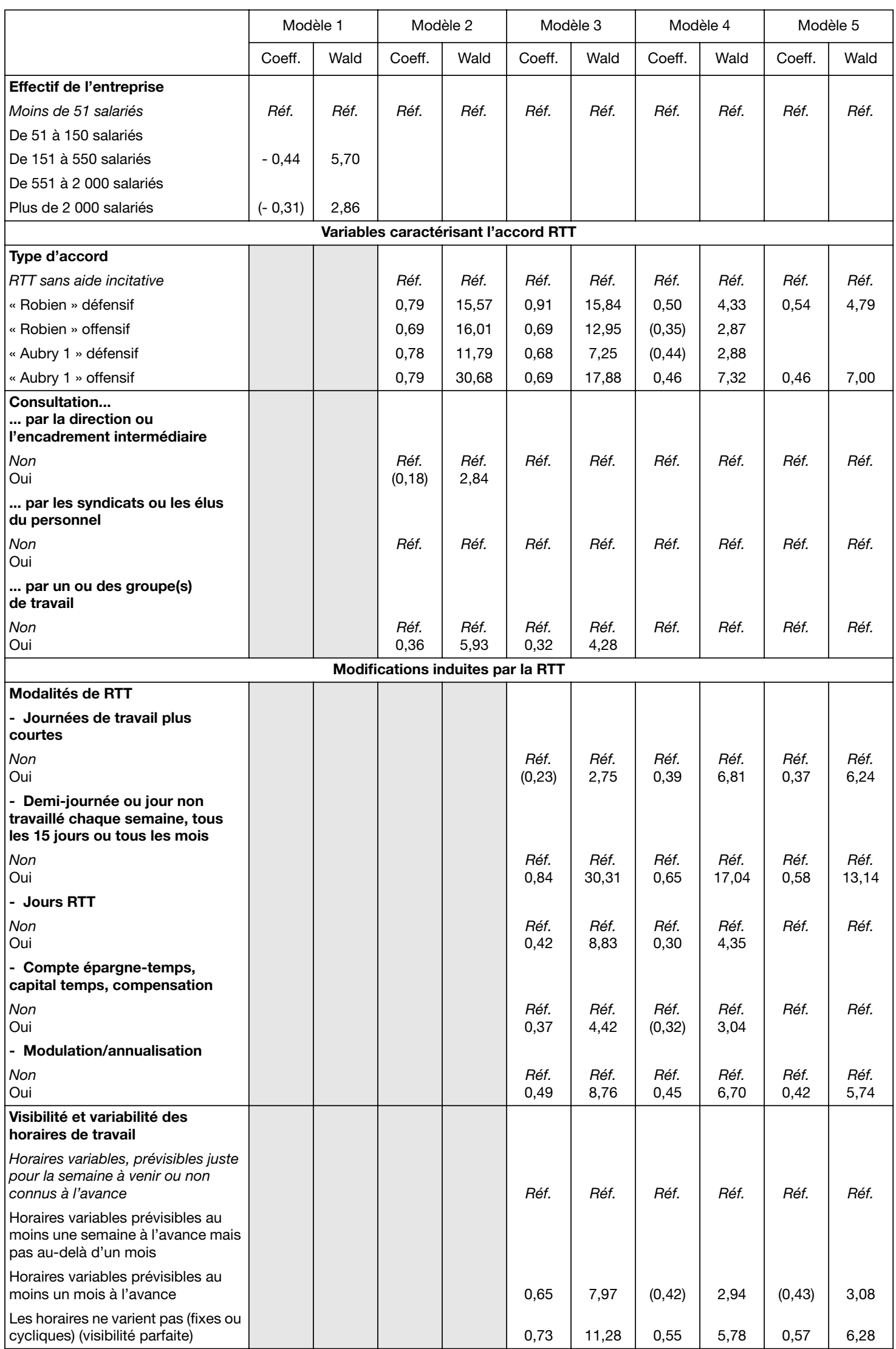


Tableau C (suite)

Approche polytomique distinguant l'amélioration, l'absence de changement et la dégradation (résultats détaillés des estimations logistiques)

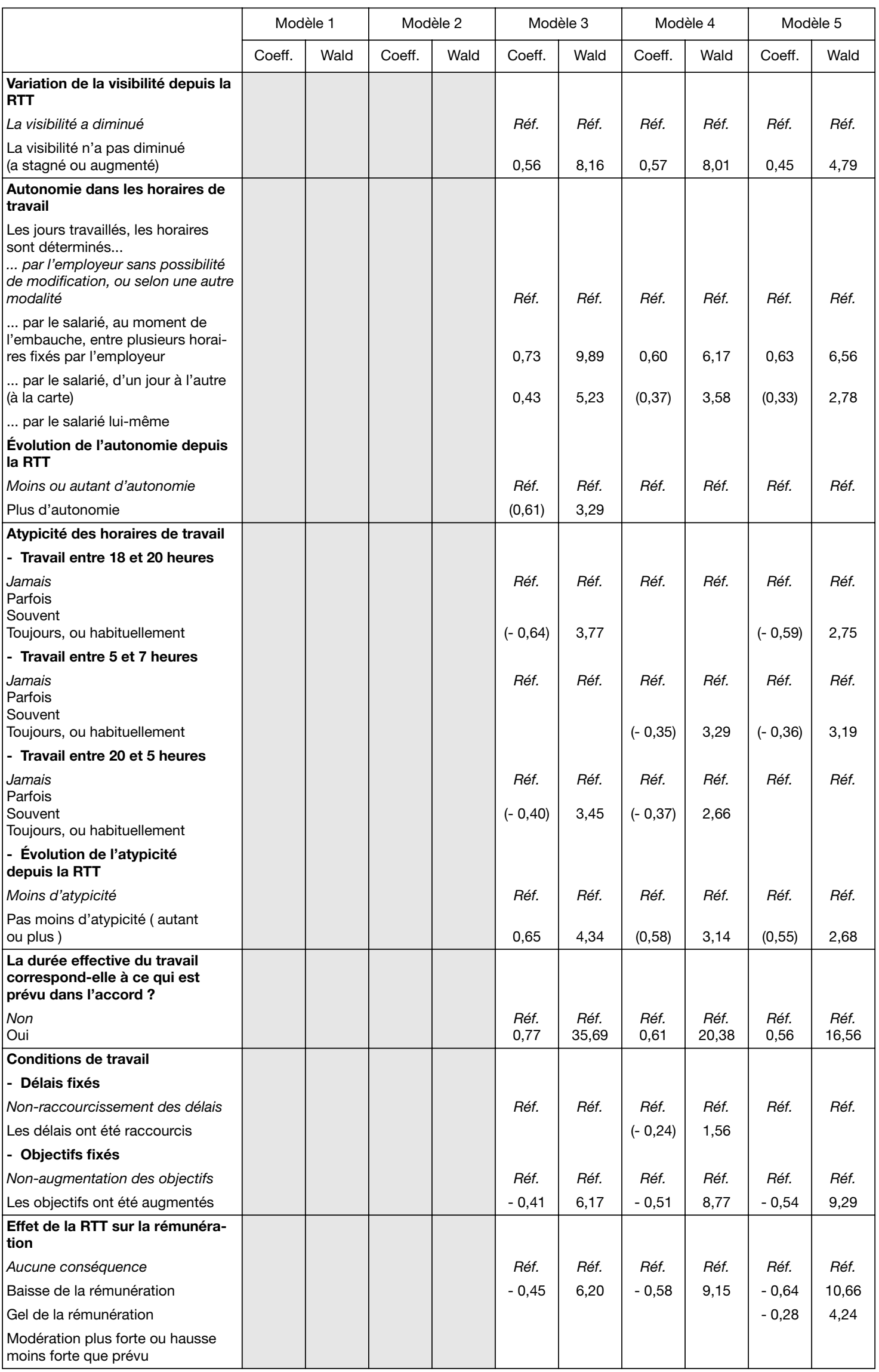


Tableau C (fin)

Approche polytomique distinguant l'amélioration, l'absence de changement et la dégradation (résultats détaillés des estimations logistiques)

\begin{tabular}{|c|c|c|c|c|c|c|c|c|c|c|}
\hline & \multicolumn{2}{|c|}{ Modèle 1} & \multicolumn{2}{|c|}{ Modèle 2} & \multicolumn{2}{|c|}{ Modèle 3} & \multicolumn{2}{|c|}{ Modèle 4} & \multicolumn{2}{|c|}{ Modèle 5} \\
\hline & Coeff. & Wald & Coeff. & Wald & Coeff. & Wald & Coeff. & Wald & Coeff. & Wald \\
\hline \multicolumn{11}{|c|}{ Usages du temps dégagé par la RTT } \\
\hline \multicolumn{11}{|l|}{$\begin{array}{l}\text { Passer plus de temps avec sa } \\
\text { famille }\end{array}$} \\
\hline 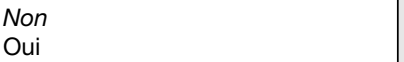 & & & & & & & $\begin{array}{l}\text { Réf. } \\
1,14\end{array}$ & $\begin{array}{l}\text { Réf. } \\
77,86\end{array}$ & $\begin{array}{l}\text { Réf. } \\
1,01\end{array}$ & $\begin{array}{l}\text { Réf. } \\
58,51\end{array}$ \\
\hline \multicolumn{11}{|l|}{$\begin{array}{l}\text { Activités domestiques, dont : } \\
\text { - Faire plus de jardinage ou de } \\
\text { bricolage }\end{array}$} \\
\hline $\begin{array}{l}\text { Non } \\
\text { Oui } \\
\text { - Passer plus de temps à au } \\
\text { moins une autre activité domesti- } \\
\text { ques }\end{array}$ & & & & & & & $\begin{array}{l}\text { Réf. } \\
(0,36)\end{array}$ & $\begin{array}{l}\text { Réf. } \\
3,68\end{array}$ & Réf. & Réf. \\
\hline $\begin{array}{l}\text { Non } \\
\text { Oui }\end{array}$ & & & & & & & $\begin{array}{l}\text { Réf. } \\
0,57\end{array}$ & $\begin{array}{l}\text { Réf. } \\
7,37\end{array}$ & $\begin{array}{l}\text { Réf. } \\
0,46\end{array}$ & $\begin{array}{l}\text { Réf. } \\
4,79\end{array}$ \\
\hline \multicolumn{11}{|l|}{$\begin{array}{l}\text { - Passer plus de temps à se } \\
\text { reposer }\end{array}$} \\
\hline $\begin{array}{l}\text { Non } \\
\text { Oui } \\
\text { - Passer davantage de temps à } \\
\text { au moins une activité de loisir } \\
\text { autre que le repos }\end{array}$ & & & & & & & $\begin{array}{l}\text { Réf. } \\
0,41\end{array}$ & $\begin{array}{l}\text { Réf. } \\
9,28\end{array}$ & $\begin{array}{l}\text { Réf. } \\
0,37\end{array}$ & $\begin{array}{l}\text { Réf. } \\
7,36\end{array}$ \\
\hline 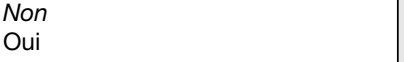 & & & & & & & $\begin{array}{l}\text { Réf. } \\
0,60\end{array}$ & $\begin{array}{l}\text { Réf. } \\
22,79\end{array}$ & $\begin{array}{l}\text { Réf. } \\
0,54\end{array}$ & $\begin{array}{l}\text { Réf. } \\
17,80\end{array}$ \\
\hline \multicolumn{11}{|c|}{ Questions « subjectives " concernant la période précédant la RTT } \\
\hline \multicolumn{11}{|l|}{$\begin{array}{l}\text { Réflexion sur l'utilisation du } \\
\text { temps }\end{array}$} \\
\hline N'avait pas réfléchi & & & & & & & & & Réf & Réf. \\
\hline $\begin{array}{l}\text { Avait réfléchi mais n'a pas utilisé le } \\
\text { temps comme souhaité }\end{array}$ & & & & & & & & & $-0,76$ & 19,01 \\
\hline $\begin{array}{l}\text { Avait réfléchi et a utilisé le temps } \\
\text { comme souhaité }\end{array}$ & & & & & & & & & 0,66 & 18,42 \\
\hline $\begin{array}{l}\text { Impression de manquer de temps } \\
\text { Jamais }\end{array}$ & & & & & & & & & & Réf. \\
\hline Rarement & & & & & & & & & $(0,33)$ & 2,77 \\
\hline Parfois & & & & & & & & & 0,34 & 4,97 \\
\hline Souvent & & & & & & & & & 0,82 & 21,46 \\
\hline Toujours & & & & & & & & & 0,53 & 8,22 \\
\hline Constante dégradation & Réf. & Réf. & Réf. & Réf. & Réf. & Réf. & Réf. & Réf. & Réf. & Réf. \\
\hline Constante statu quo & & & $-1,18$ & 9,21 & $-2,91$ & 31,48 & $-2,85$ & 27,76 & $-3,07$ & 30,41 \\
\hline Constante amélioration & 1,34 & 13,84 & & & 1,07 & 4,35 & & & $(-0,96)$ & 3,00 \\
\hline Nombre d'observations & & & & & & & & & & \\
\hline P-value LR & & 001 & $<0$ & & & & $<0$ & 001 & $<0$, & 001 \\
\hline P-value Score & & 001 & $<0$ & & & & $<0$ & 001 & $<0$, & 001 \\
\hline P-value Wald & & 001 & $<0$ & & & 001 & $<0$ & 001 & $<0$, & 001 \\
\hline P-value test d'égalité des pentes & & & & & & 001 & $<0$ & 001 & $<0$, & 001 \\
\hline Concordance & & & & & & & & & & \\
\hline
\end{tabular}

Les situations de référence dans les estimations sont indiquées par « Réf. ».

Les 5 modèles se distinguent par un nombre croissant de variables explicatives retenues. Ainsi, les variables explicatives retenues sont : dans le modèle 1, les seules variables d'état; dans le modèle 2, les variables explicatives du modèle 1 et les variables caractérisant l'accord; dans le modèle 3, les variables explicatives du modèle 2 et les variables indiquant les modifications induites par la RTT; dans le modèle 4, les variables explicatives du modèle 3 et les variables indiquant les usages du temps dégagé par la RTT; dans le modèle 5, les variables explicatives du modèle 4 et les variables de réponses aux questions "subjectives " concernant la période précédant l'accord.

La statistique de Wald est le carré de la statistique de Student. Si elle dépasse 4, cela signifie que le coefficient est significatif au seuil de $5 \%$. Les coefficients non significatifs ne sont pas indiqués afin d'alléger le tableau. Mais les coefficients proches (à un seuil compris entre $5 \%$ et $10 \%)$ sont cependant fournis entre parenthèses.

Lecture : dans le cadre du modèle 2, pour la rubrique "Type d'accord " les quatre modalités se distinguent significativement au seuil de $5 \%$ des $R T T$ sans aide incitative retenus comme référence. Les salariés présentant un accord de RTT avec aide incitative ont ainsi une probabilité d'avoir connu, via la RTT, une amélioration de leur vie quotidienne significativement supérieure à ceux dont l'accord de RTT ne s'accompagne pas d'une telle aide.

Source des données de base : enquête RTT et Modes de vie, Dares. 